\title{
Deep Graph Pose: a semi-supervised deep graphical model for improved animal pose tracking
}

\author{
Anqi Wu ${ }^{1 *} \quad$ E. Kelly Buchanan ${ }^{1 *} \quad$ Matthew R Whiteway $^{1} \quad$ Michael Schartner $^{2}$ \\ Guido Meijer $^{3} \quad$ Jean-Paul Noel $^{4} \quad$ Erica Rodriguez $^{1} \quad$ Claire Everett $^{1}$ \\ Amy Norovich $^{1} \quad$ Evan Schaffer $^{1} \quad$ Neeli Mishra $^{1} \quad$ C. Daniel Salzman ${ }^{1}$ \\ Dora Angelaki $^{4} \quad$ Andrés Bendesky $^{1} \quad$ The International Brain Laboratory $^{5}$ \\ John Cunningham ${ }^{1} \quad$ Liam Paninski ${ }^{1}$ \\ ${ }^{1}$ Columbia University, New York, USA \\ \{aw3236, ekb2154, mw3323, er2934, cpe2108, aln2128, ess2129, nm2786 \\ cds2005, ab4463, jpc2181, lmp2107\}@columbia.edu \\ ${ }^{2}$ University of Geneva, Geneva, Switzerland \\ Michael.Schartner@unige.ch \\ 3 The Champalimaud Centre for the Unknown, Lisbon, Portugal \\ guido.meijer@research.fchampalimaud.org \\ ${ }^{4}$ New York University, New York, USA \\ \{jpn5, da93\}@nyu.edu \\ 5 info@internationalbrainlab.org
}

\begin{abstract}
Noninvasive behavioral tracking of animals is crucial for many scientific investigations. Recent transfer learning approaches for behavioral tracking have considerably advanced the state of the art. Typically these methods treat each video frame and each object to be tracked independently. In this work, we improve on these methods (particularly in the regime of few training labels) by leveraging the rich spatiotemporal structures pervasive in behavioral video - specifically, the spatial statistics imposed by physical constraints (e.g., paw to elbow distance), and the temporal statistics imposed by smoothness from frame to frame. We propose a probabilistic graphical model built on top of deep neural networks, Deep Graph Pose (DGP), to leverage these useful spatial and temporal constraints, and develop an efficient structured variational approach to perform inference in this model. The resulting semi-supervised model exploits both labeled and unlabeled frames to achieve significantly more accurate and robust tracking while requiring users to label fewer training frames. In turn, these tracking improvements enhance performance on downstream applications, including robust unsupervised segmentation of behavioral "syllables," and estimation of interpretable "disentangled" low-dimensional representations of the full behavioral video. Open source code is available at https://github.com/paninski-lab/deepgraphpose.
\end{abstract}

\footnotetext{
*equal contribution
} 


\section{Introduction}

Animal pose estimation (APE) is a critical scientific task, with applications in ethology, psychology, neuroscience, and other fields. Recent work in neuroscience, for example, has emphasized the degree to which neural activity throughout the brain is correlated with movement $[1,2,3]$; i.e., to understand the brains of behaving animals we need to extract as much information as possible from behavioral video recordings. State of the art APE methods, such as DeepLabCut (DLC) [4], DeepPoseKit (DPK) [5], and LEAP [6], have transferred tools from human pose estimation (HPE) in deep learning literature to the APE setting [7,8], opening up an exciting array of new applications and new scientific questions to be addressed.

However, even with these advances in place, hundreds of labels may still be needed to achieve tracking at the desired level of precision and reliability. Providing these labels requires significant user effort, particularly in the common case that users want to track multiple objects per frame (e.g., all the fingers on a hand or paw). Unlike HPE algorithms [9], APE algorithms are applied to a wide variety of different body structures (e.g., fish, flies, mice, or cheetahs) [10], compounding the effort required to collect labeled datasets and hindering our ability to re-use a common skeletal model. Moreover, even with hundreds of labels, users still often see occasional "glitches" in the output (i.e., frames where tracking is briefly lost), which typically interfere with downstream analyses of the extracted behavior.

To improve APE performance in the sparse-labeled-data regime, we propose a probabilistic graphical model built on top of deep neural networks, Deep Graph Pose (DGP), to leverage both spatial and temporal constraints, and develop an efficient structured variational approach to perform inference in this model. DGP is a semi-supervised model that takes advantage of both labeled and unlabeled frames to achieve significantly more accurate and robust tracking, using fewer labels. Finally, we demonstrate that these tracking improvements enhance performance in downstream applications, including robust unsupervised segmentation of behavioral "syllables," and estimation of interpretable low-dimensional representations of the full behavioral video.

\section{Related Work}

Animal pose estimation. The proposed approach fills a void between state of the art human pose estimation algorithms, which often rely on large quantities of manually labeled samples (see [9] for a recent review), and their counterparts in animal pose estimation [11, 4, 6, 5, 12, 13]. Among these animal pose estimation algorithms, DLC [4], LEAP [6], and DPK [5] stand out as they can achieve near human-level accuracy. However, all these methods rely on a large number of human labels in order to achieve the desired level of precision and reliability. Our work extends such models with a probabilistic graphical model that use unlabeled frames and temporal and spatial structures. [14] has recently proposed to incorporate temporal context from nearby video frames using optical flow which occurs only at the test stage to refine the model's predictions. However, in our approach, we incorporate the temporal context into the trainable graphical model.

Graphical models. Previous work on human pose estimation has employed graphical models as regularizers for convolutional networks [15, 16, 17, 18, 19, 20]. Among these, [17] and [18], like DGP, build an undirected graphical model (UGM) on top of deep neural networks. However, unlike DGP, they assign tracked locations discrete values, which allows for (discrete) message passing algorithms during the inference step. [19] builds a spatial-temporal graph similar to DGP. But none of these previous methods uses unlabeled frames to improve performance, as DGP does. They were all proposed for human pose estimation which has many benchmark datasets with a large number of labels. [20] has proposed a method later for sparsely-labeled videos but without any spatial constraints.

Semi-supervised learning. Semi-supervised learning aims to fully utilize unlabeled or weaklylabeled data to gain additional insights into the structure of the data [21, 22, 23]. Many pose estimation algorithms have adopted such learning schemes to enhance the performance given limited training data $[24,25]$. One conceptually similar "weakly-supervised" approach is described by [26], who trained a network to extract flying objects (obeying Newtonian acceleration) simply by constraining the output to resemble a parabola. In our work, DGP encourages the output confidence 


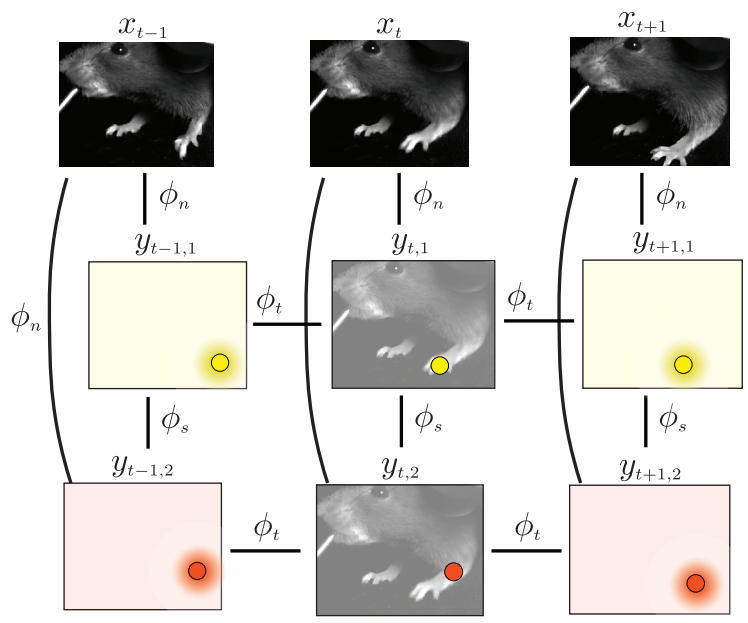

Figure 1: Deep Graph Pose (DGP) model. DGP leverages observed (labeled) and hidden information to infer the locations of unobserved targets via graph semisupervised inference. At each time $t$, we observe the frame $x_{t}$. We want to track multiple targets in each frame (in this case, the paw and elbow). We also observe the labels of the two targets in some frames (in this example, in the $t$-th frame), denoted as $y_{t, 1}$ and $y_{t, 2}$ (colored circles at $t$ ). The hidden variables are the unobserved targets (indicated with colored circles in the colored background in frames $t-1$ and $t+1$ here).

map to be unimodal; this can be seen as a form of weak supervision that leads to improved accuracy even when the temporal and spatial soft constraints are removed.

\section{Model}

The graphical model of DGP is summarized in Figure 1. We observe frames $x_{t}$ indexed by $t$, along with a small subset of labeled markers $y_{t, j}$ (where $j$ indexes the different targets we would like to track). The target locations $y_{t, j}$ on most frames are unlabeled, but we have several sources of information to constrain these latent variables: temporal smoothness constraints between the targets $y_{t, j}$ and $y_{t+1, j}$, which we capture with potentials $\phi_{t}$; spatial constraints between the targets $y_{t, i}$ and $y_{t, j}$, which we model with spatial potentials $\phi_{s}$; and information from the image $x_{t}$, modeled by $\phi_{n}$.

We parametrize $\phi_{n}$ with a neural network, indicated by the subscript $n$. A number of architectures could potentially be employed for $\phi_{n}[6,5]$; we chose to adapt the architecture used in DLC [4] here.

For simplicity, we start with a quadratic potential $\phi_{t}$ to impose temporal smoothness:

$$
\phi_{t}^{j}\left(y_{t, j}, y_{t+1, j}\right)=\frac{1}{2} w_{t}^{j}\left\|y_{t, j}-y_{t+1, j}\right\|^{2},
$$

which penalizes the distance between targets in consecutive frames; the weights $w_{t}^{j}$ in general may depend on the target index $j$, and can also vary in time. A quadratic potential is equivalent to modeling the target at the next time step as normally distributed around the current target, which is also equivalent to Gaussian random walk. We will discuss extensions of this simple quadratic potential in the appendix.

The spatial potential $\phi_{s}$ is more dataset-dependent and can be chosen depending on the constraints that the markers should satisfy. Typical examples include a soft constraint that the paw marker should not exceed some distance from the elbow marker, or the nose should always stay within a certain radius of a static waterspout. Again, we start with a simple quadratic potential to encode these soft constraints:

$$
\phi_{s}^{i j}\left(y_{t, i}, y_{t, j}\right)=\frac{1}{2} w_{s}^{i j}\left\|y_{t, i}-y_{t, j}\right\|^{2},
$$

which penalizes the distance between "connected" targets $y_{t, i}$ and $y_{t, j}$ (where the user can pre-specify pairs of connected targets that should have neighboring locations in the frame, e.g. paw and elbow); more sophisticated non-quadratic losses are again discussed in the appendix.

We want to "let the data speak" and avoid oversmoothing, so the penalty weights $w_{s}$ and $w_{t}$ should be small. In practice we found that the temporal weights $w_{t}^{j}$ could be set using optical flow [27] which captures the vector field between adjacent frames. We first computed the vector field between two neighbor frames $t-1$ and $t$ using optical flow. Then we calculated the average motion vector for target $j$ from frame $t-1$ to frame $t$. The magnitude of the motion vector was denoted as $m_{t}^{j}$. Finally $w_{t}^{j}=\xi / m_{t}^{j}$, where $\xi$ is a constant scalar independent of dataset, time and target indices. The 
intuition is the larger the movement of the target is, the smaller the temporal clique weight should be. We set the spatial weights as $w_{s}^{i j}=c / d_{i j}$, where $d_{i j}$ is a rough estimate of the average distance (in pixels) between targets $i$ and $j$ and $c>0$ is a small scalar (again independent of dataset and target indices $i, j$ ), which led to robust results without any need to fit extra parameters. We summarize the parameter vector as $\beta=\left\{\theta, w_{t}, w_{s}\right\}$, where $\theta$ denotes the neural net parameters in $\phi_{n}$. Given $\beta$, the joint probability distribution over targets $y$ is

$$
\begin{aligned}
p(y \mid x, \beta)=\frac{1}{Z(x, \beta)} \exp ( & -\sum_{t=1}^{T} \sum_{j=1}^{J} \phi_{n}^{j}\left(y_{t, j}, x_{t}\right) \\
& \left.-\sum_{t=1}^{T-1} \sum_{j=1}^{J} \phi_{t}^{j}\left(y_{t, j}, y_{t+1, j}\right)-\sum_{t=1}^{T} \sum_{i, j \in \mathcal{E}} \phi_{s}^{i j}\left(y_{t, i}, y_{t, j}\right)\right),
\end{aligned}
$$

where $\mathcal{E}$ denotes the edge set of constrained targets (i.e., the pairs of markers $i, j$ with a nonzero potential function), $Z(x, \beta)=\int p(y \mid x, \beta) d y$ is the normalizing constant marginalizing out $y, T$ denotes the total number of frames, and $J$ denotes the total number of targets.

\section{Structured variational inference}

Our goal is to estimate $p\left(y^{h} \mid y^{v}, x, \beta\right)$, the posterior over locations of unlabeled targets $y^{h}$, given the frames from the video $x$, the locations of the labeled markers $y^{v}$, and the parameters $\beta$. Here $h$ denotes hidden, for the unlabeled data, and $v$ denotes visible, for the labeled data. Calculating this posterior distribution exactly is intractable, due to the highly nonlinear convolutional networks appearing in potentials $\phi_{n}$. We chose to use structured variational inference [28, 29] to approximate this posterior. We approximate $p\left(y^{h}, y^{v} \mid x, \beta\right)$ with a Gaussian graphical model (GGM) with the same graphical model as Figure 1, leading to a Gaussian posterior approximation $q\left(y^{h} \mid y^{v}, x, \beta\right)$ for $p\left(y^{h} \mid y^{v}, x, \beta\right)$ in which the inverse covariance (precision) matrix is block tridiagonal (Gaussian random walk), with one block per frame $t$. Since the potentials $\phi_{t}$ and $\phi_{s}$ are quadratic, yielding Gaussian distributions, the neural-network image potential $\phi_{n}$ is the only term that needs to be replaced with a new quadratic potential to form a Gaussian $q$.

Updating the parameters of this GGM scales as $O\left(T J^{3}\right)$ in the worst case, due to the chain structure of the graphical model (and the corresponding block tridiagonal structure of the precision matrix). If the edge graph $\mathcal{E}$ defined by the user-specified spatial potential function set is disconnected, this $J^{3}$ factor can be replaced by $K^{3}$, where $K$ is the size of the largest connected component in $\mathcal{E}$.

We used a structured inference network approach [29] to estimate the model and variational parameters. We computed gradients of the evidence lower bound (ELBO) for this model using standard automatic differentiation tools, and performed standard stochastic gradient updates to estimate the parameters. Full details regarding the ELBO derivation and optimization can be found in Section S1 in the appendix.

\subsection{Conceptual comparison against fully-supervised approaches}

Standard fully-supervised approaches like DeepLabCut [4] learn a neural network (or more precisely, use transfer learning to adjust the parameters of an existing neural network) to essentially perform a classification task: the network is trained to output large values at the known location of the markers (i.e., the "positive" training examples), and small values everywhere else (the "negative" training examples). Given a small number of training examples, these methods are prone to overfitting.

In contrast, the approach we propose here is semi-supervised: it takes advantage of both the labeled and unlabeled frames to learn better model parameters $\theta$. On labeled frames, the posterior distribution $p\left(y^{v} \mid y^{v}, x, \beta\right)$ is deterministic, and the objective function reduces to the fully supervised case. On the other hand, on unlabeled frames we have new terms in the objective function (see section S1.2.1 for more details). Clearly, the spatial and temporal potentials $\phi_{s}$ and $\phi_{t}$ encourage the outputs to be temporally smooth and to obey the user-specified spatial constraints (at least on average). But in addition the objective function encourages $\phi_{n}$ to output large values where $p\left(y^{h} \mid y^{v}, x, \beta\right)$ is large, and small values where $p\left(y^{h} \mid y^{v}, x, \beta\right)$ is small. Since we approximate $p\left(y^{h} \mid y^{v}, x, \beta\right)$ as Gaussian, the resulting ELBO encourages $\phi_{n}$ to be (on average) unimodal on unlabeled frames - a constraint 
Table 1: Dataset summary.

\begin{tabular}{lccc}
\hline \multicolumn{1}{c}{ Dataset } & $\begin{array}{c}\text { Brief } \\
\text { Description }\end{array}$ & $\begin{array}{c}\text { Dimensions } \\
(\mathrm{x}, \mathrm{y}, \mathrm{t})\end{array}$ & $\begin{array}{c}\text { Number of } \\
\text { labeled frames }\end{array}$ \\
\hline mouse-wheel [30] & moving a wheel & $(374,450,1000)$ & 55 \\
mouse-reach [31] & grabbing a stick & $(747,832,256)$ & 52 \\
fly-run [32] & running on a ball & $(600,600,1210)$ & 13 \\
twomice-top-down* & freely moving & $(480,640,1364)$ & 20 \\
fish-swim [33] & freely swimming & $(471,475,2000)$ & 20 \\
\hline
\end{tabular}

(*) unpublished
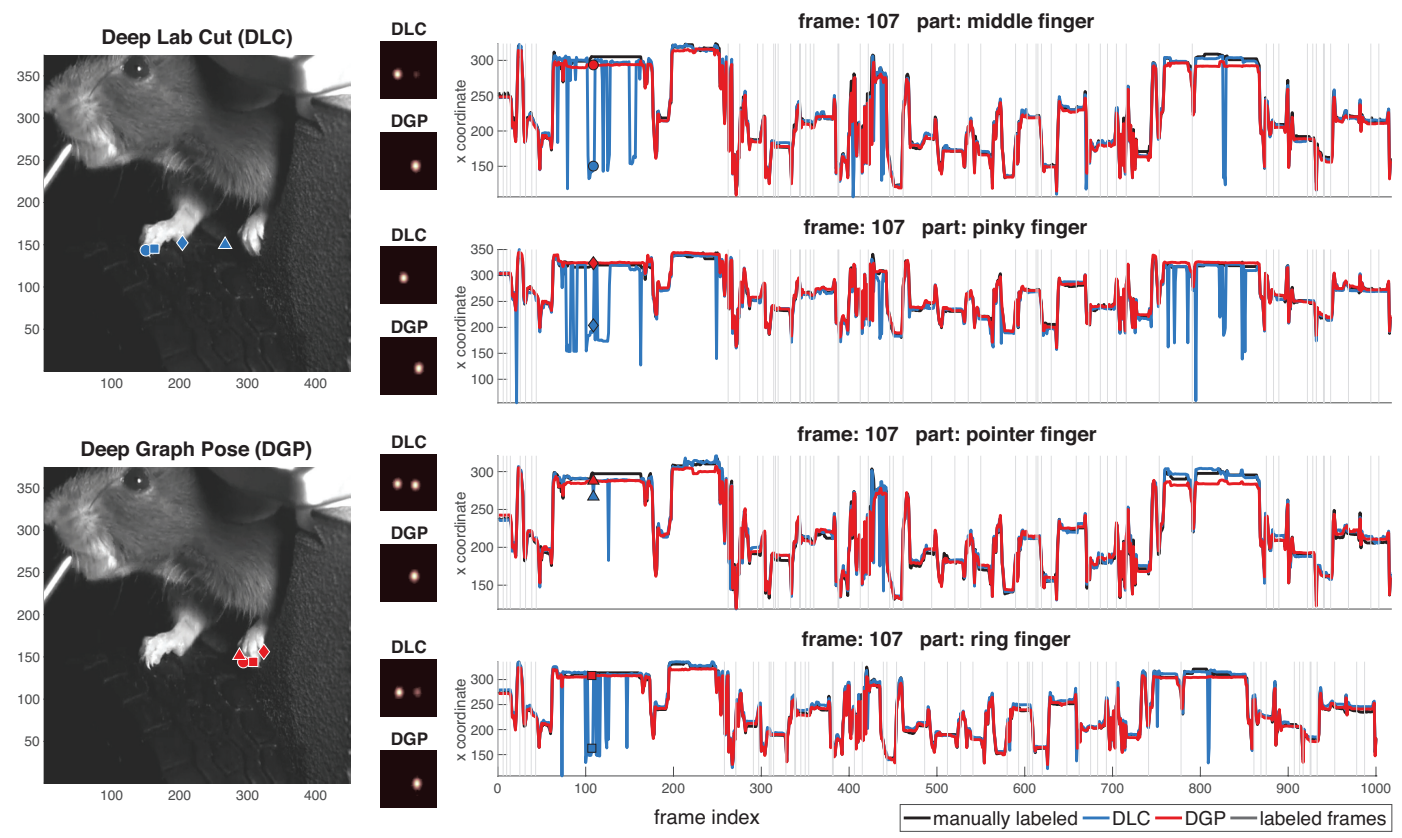

Figure 2: Comparison of Deep Graph Pose (DGP) versus DeepLabCut (DLC) and manuallylabeled data on the mouse-wheel dataset from [30]; see also [34]. Left panels show an example frame, with the DLC output markers superimposed in blue (top) and the DGP markers in red (bottom). The right panels show the horizontal marker positions as a function of time (with DLC in blue, DGP in red and the full manually-labeled trace in black). Vertical lines indicate labeled (training) frames. The small inset images show confidence maps for each marker output by DLC (top) and DGP (bottom); the DGP confidence maps tend to be more unimodal than the DLC confidence maps. Note that the DLC and DGP marker locations tend to agree on labeled frames, but we see significant discrepancies on unlabeled test frames. Visual inspection of the videos (and comparison again the manual labels) indicates that when the DLC and DGP markers disagree, typically the DLC marker is in the wrong location.

that is not enforced in standard approaches. This turns out to be a powerful regularizer and can lead to significant improvements even in cases where the spatial and temporal constraints $\phi_{s}$ and $\phi_{t}$ are weak, as we will see in the next section.

\section{Results}

We applied DGP and DLC ${ }^{2}$ to a variety of datasets, including behavioral videos from three different species, in a variety of poses and environments (see Table 1 for a summary). The new model (DGP) consistently outperformed the baseline (DLC). In each example video analyzed here, DLC outputs

\footnotetext{
${ }^{2}$ https://github.com/AlexEMG/DeepLabCut
} 


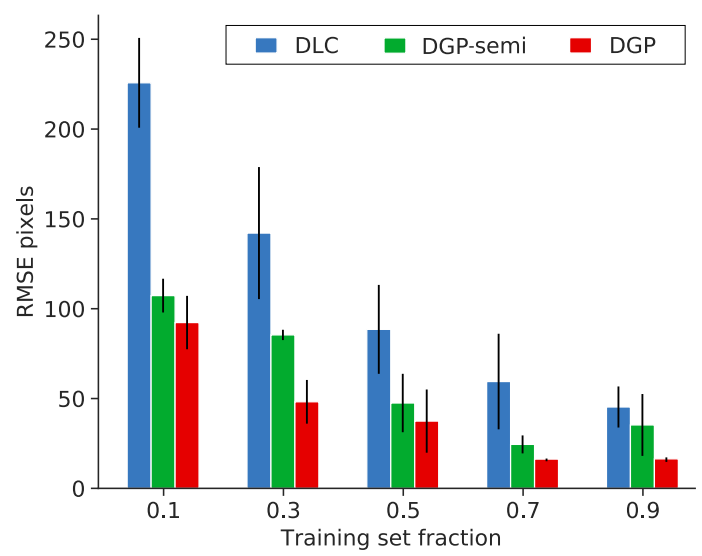

Figure 3: Quantification of the results from Figure 2 over multiple training set sizes and ablation experiments. DGP outperforms DLC and the intermediate variant DGP-semi. We evaluated the different methods (see main text for definition of DGP-semi) using multiple random subsets of the training set (55 labels) and compared the differences in test error. Error bars represent one standard error across five random trials. Each random trial has its own randomly generated training set.

occasional "glitch" frames where tracking of at least one target was lost (e.g., around frame index 100 in the lower right panel); these glitches were much less prevalent in the DGP output. We experimented with running Kalman smoothers and total variation denoisers to post-process the DLC output, but were unable to find any parameter settings that could reliably remove these glitches without oversmoothing the data (results not shown). The frequency of these "glitches" can be reduced by increasing the training set through labeling more data - but this is precisely the user effort we aim to minimize here. See the full videos summarizing the performance of the two methods. An example screenshot for the mouse-wheel dataset [30] is shown in Figure 2. The comparison between DLC and DGP on all other datasets can be found in Figures S3-S6 in the appendix. More information regarding experimental setup can be found in Section S4 in the appendix.

We also examined the "confidence maps" generated by visualizing the output of the neural network $\phi_{n}$ as an image; large values of the confidence map indicated the regions where the network "believed" the target was located with high confidence. Comparing the confidence maps output by DLC versus DGP, we see that the latter tended to be more unimodal (see Figure 2, small panels in the middle column). Nonetheless, DGP did occasionally output multi-modal confidence maps (e.g., in frames where the target was occluded), since the ELBO objective function used to train DGP encouraged unimodality but did not impose unimodality as a hard constraint.

To better understand the source of the performance gains exhibited by DGP, we also experimented with a model in which the spatial and temporal potentials were turned off (i.e., $w_{s}=w_{t}=0$ ). The resulting graphical model can be factorized over targets $j$ and frames $t$. We call the resulting model DGP-semi, since the resulting ELBO objective function combines a usual supervised loss (as in DLC) with an unsupervised term that encourages the output of the image potential $\phi_{n}$ to match its Gaussian approximation for each $(t, j)$ pair (i.e., the resulting loss can be considered a semi-supervised hybrid model). Comparing DLC, DGP-semi, and DGP provides a qualitative sense of the relative benefits of the semi-supervised loss and the spatial and temporal cliques (see videos).

To develop more quantitative comparisons, we manually labeled 1000 frames in the mouse-wheel dataset $^{3}$. We randomly assigned 55 labeled frames to the training set and used the remaining 945 frames as the test set. Next we randomly subsampled $10 \%-90 \%$ of this training set and retrained the models to quantify the relation between the test errors and the number of labeled frames. Figure 3 shows the test errors averaged over five random subsamples. We see that DGP-semi and DGP outperformed DLC uniformly over the training set fractions (i.e., the number of labeled frames used to train the model) with a significant amount of improvement. DGP further decreased the errors with the extra spatial and temporal constraints. Similar results were obtained using an $\epsilon$-insensitive loss that ignored errors below a threshold $\epsilon$ (on the order of 5-10 pixels here) below which the "true" marker location becomes somewhat subjective (results not shown here).

From both qualitative and quantitative analyses, we can tell that although DGP-semi does not enforce any spatial constraints or temporal smoothness, the extra regularization from the unsupervised term

\footnotetext{
${ }^{3}$ This exhaustive labeling was labor-intensive and we have not yet performed the same analysis for the other datasets in Table 1. As is visible in the appendix figures, our qualitative results are similar across all the datasets analyzed here; we plan to perform more exhaustive comparisons on other datasets in the future.
} 
A

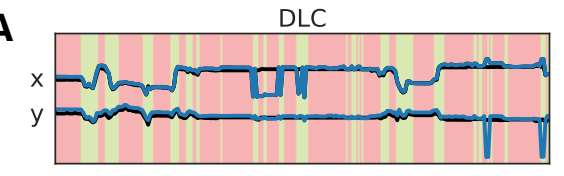

DGP

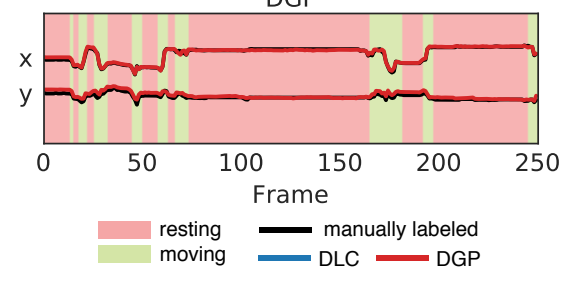

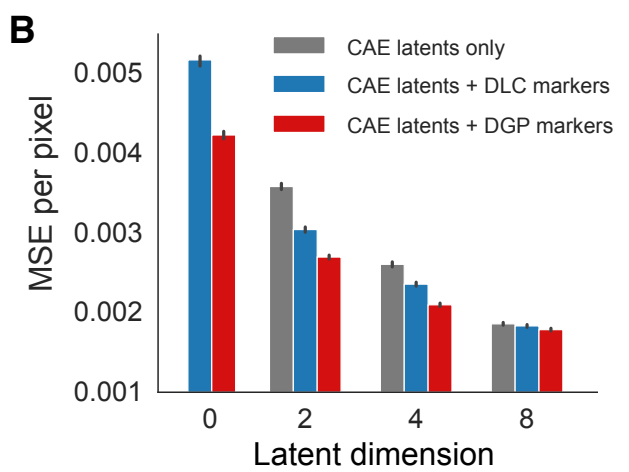

Figure 4: (A) Unsupervised methods segment DGP traces into interpretable "resting" versus "moving" states, while DLC trace segmentation is hampered by glitches. We ran a two-state autoregressive hidden Markov model (ARHMM) on the DGP and DLC outputs (in this case, on the $\mathrm{x}$ - and $\mathrm{y}$-coordinates of a single paw). Background colors indicate the inferred states from the ARHMM fit to the DGP or DLC traces. The model fit with the DGP output clearly learns interpretable states, a "resting" state (red) and a "moving" state (green) (bottom). The model fit with the DLC output learns two states that are partially corrupted by "glitches" where DLC jumps away from the manually-labeled paw position (bottom); see video for full details. (B) Conditioning CAEs on DGP markers improves reconstruction performance. We computed mean square error (MSE) per pixel on reconstructed test frames from the mouse-wheel dataset when using a CAE (gray bars), or conditional CAEs, where the markers output by DLC (blue) or DGP (red) are used as input to both the encoder and decoder networks. A latent dimension of 0 corresponds to directly decoding the frames from markers. We see that test MSE decreases with latent dimensionality (as expected), and that the model conditioned on DGP markers consistently outperforms the model conditioned on DLC markers. Error bars represent 95\% bootstrapped confidence interval over test frames. Reconstruction videos are also available.

in the ELBO encourages the model output to be more unimodal, leading to significantly improved predictions compared to DLC. With the additional temporal and spatial constraints, DGP can further improve the performance.

\subsection{Downstream analyses}

The above results demonstrate that DGP provides improved tracking performance compared to DLC. Next we show that these accuracy improvements can in turn lead to more robust and interpretable results from downstream analyses based on the tracked output.

Unsupervised temporal segmentation. We begin with a segmentation task: given the estimated trace for the paw, can we use unsupervised methods to determine, e.g., when the paw is moving versus still? Figure 4A shows that the answer is yes if we use the DGP output: a two-state auto-regressive hidden Markov model (ARHMM; fit via Gibbs sampling on 1000 frames output from either DGP or DLC; [35]) performs well with no further pre- or post-processing. In contrast, the multiple DLC "glitches" visible in Figure 2 contaminate the segmentation based on the DLC traces, resulting in unreliable segmentation. See the video for further details. Similar results were obtained when fitting models with more than two states (data not shown).

Conditional convolutional autoencoder (CAE) for more interpretable low-dimensional representation learning. As a second downstream application, we consider unsupervised dimensionality reduction of behavioral videos [3, 1, 36, 37]. This approach, which typically uses linear methods like singular value decomposition (SVD), or nonlinear methods like convolutional autoencoders (CAEs), does not require user effort to label video frames. However, interpreting the latent features of these models can be difficult [38, 39], limiting the scientific insight gained by using these models. A hybrid approach that combines supervised (or semi-supervised) object tracking with unsupervised CAE training has the potential to ameliorate this problem [40, 41, 42, 43] - the tracked targets encode information about the location of specific body parts, while the estimated CAE latent vectors encode the remaining sources of variability in the frames. We refer to this ideal partitioning of variability into 

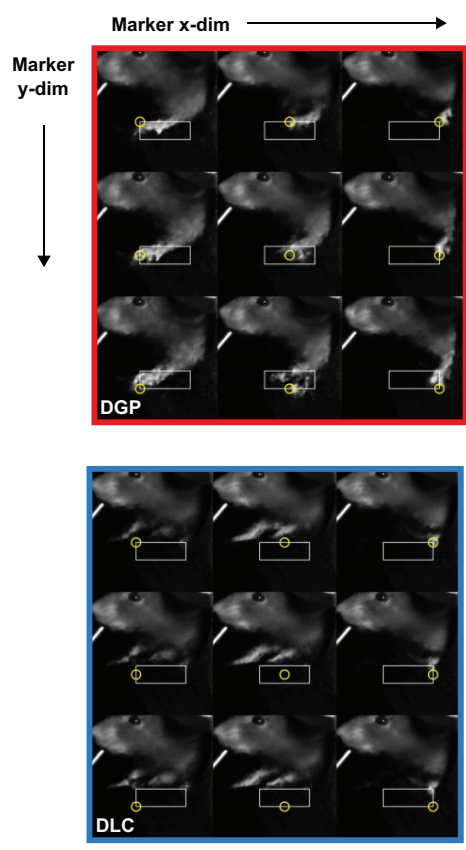

Manipulating markers AE latents fixed
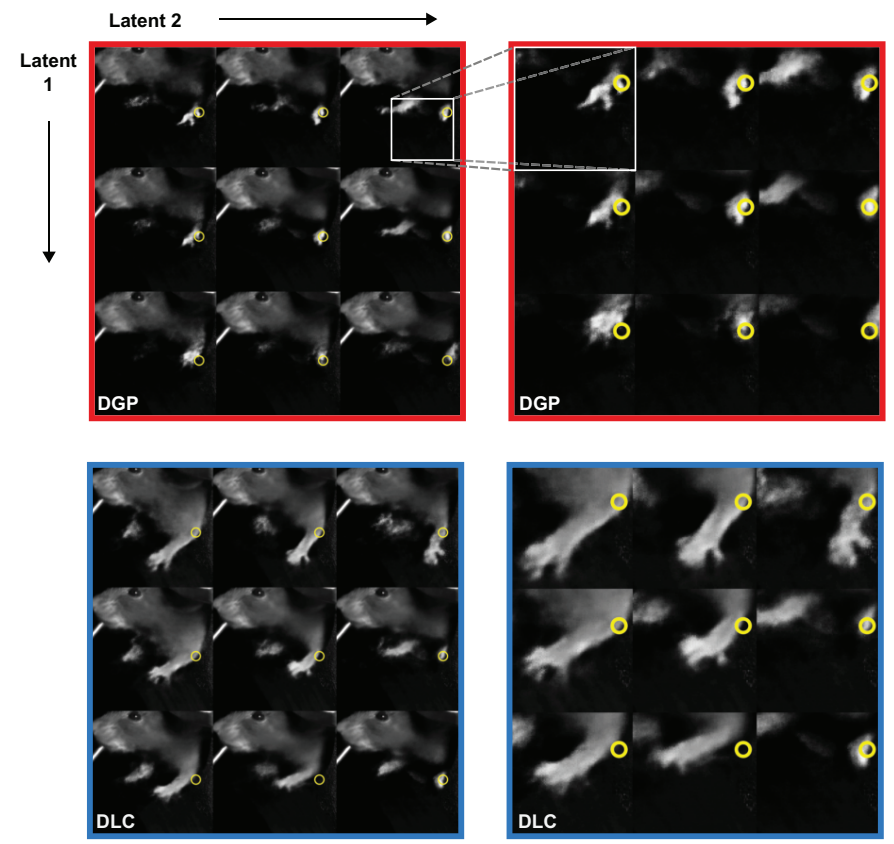

Manipulating AE latents Markers fixed

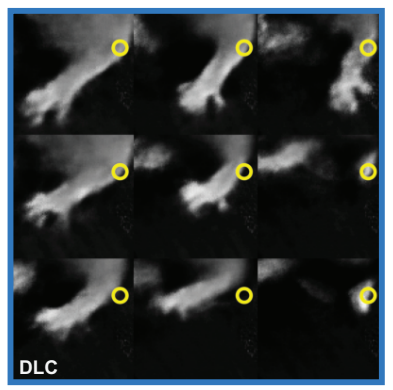

Manipulating AE latents Markers fixed (paw zoom)

Figure 5: Conditioning CAEs on DGP markers, but not DLC markers, leads to disentangled latents. We incorporated the DLC and DGP markers into conditional CAEs trained on the mousewheel dataset. All frames are generated from 2-latent networks. Left: frames generated from the CAEs when changing the $\mathrm{x}$ and $\mathrm{y}$ coordinates of the left paw marker (yellow circle) for a given frame, with all other latents/markers fixed (white bounding box denotes the range of $\mathrm{x} / \mathrm{y}$ coordinates). This manipulation should lead to noticeable changes in left paw position if markers are disentangled from latents. The network trained with DGP markers affords a much higher degree of control and produces more realistic looking images than that trained with DLC. Center: frames generated from the CAEs when changing the latents, with all markers fixed (white bounding box denotes the crop used for the right panels). This manipulation should not change the left paw position, but rather vary other (untracked) features of the image. Changes in the DGP reconstructions are limited to a small region around the tracked paws (yellow circle denotes left paw marker; see right panels for crop), demonstrating that the latents are encoding more local information such as paw configuration. DLC reconstructions show undesirable large movements of the left paw, demonstrating that the latents are encoding information about this tracked body part that should be present in the markers. Right: zoom of cropped region around the original paw location for frames in the center panel. See appendix Figure S1 for a more detailed quantitative analysis of latent/marker disentanglement.

more interpretable subspaces as "disentangling." Below we show that these hybrid models produce features that are more disentangled when trained with the output from DGP compared to DLC.

We fit conditional CAEs that take the markers output by DLC or DGP (hereafter referred to as CAE-DLC and CAE-DGP, respectively) as conditional inputs into both the encoding and decoding networks of the CAE, using the mouse-wheel dataset with 13 randomly chosen labeled frames (see Section S2 for implementation details). For this analysis, to obtain useful information across the full image, we labeled the left paw, right paw, tongue, and nose, rather than the four fingers on the left paw as in the previous section. Incorporating the tracking output from either method decreases the mean square error (MSE) of reconstructed test frames, for a given number of latents (Figure 4B). Furthermore, the networks trained with DGP outputs show improved performance over those trained with DLC outputs. Subsequent analyses are performed on the 2-latent networks, for easier visualization. 
To test the degree of disentanglement between the CAE latents and the DGP or DLC output markers, we performed two different manipulations. First, we asked how changing individual markers affects the CAE reconstructions. We manipulate the $\mathrm{x} / \mathrm{y}$ coordinates of a single marker while holding all other markers and all latents fixed. If the markers are disentangled from the latents we would expect to see the body part corresponding to the chosen marker move around the image, while all other features remain constant. We randomly chose a test frame and simultaneously varied the $\mathrm{x} / \mathrm{y}$ marker values of the left paw (Figure 5, left). This manipulation results in realistic looking frames with clear paw movements in the CAE-DGP reconstructions, demonstrating that this marker information has been incorporated into the decoder. For the CAE-DLC reconstructions, however, this manipulation does not lead to clear movements of the left paw, indicating that the decoder has not learned to use these markers as effectively (a claim which is also supported by the higher MSE in the CAE-DLC networks, Figure 4B).

Second, we asked how changing the latents (rather than markers) affects the reconstructed frames. In this manipulation we simultaneously change the values of the two latents while holding all markers fixed. If the latents are disentangled from the markers we expect to see the tracked features remain constant while other untracked features change. For the CAE-DGP network this latent manipulation has very little effect on the tracked body parts, as desired (Figure 5, top center); instead, the manipulation leads to small changes in the configuration of the left paw (rather than its absolute location; Figure 5, top right). On the other hand, for the CAE-DLC network this latent manipulation has a large effect on the left paw location (Figure 5, bottom center), which should instead be encoded by the markers. These results qualitatively demonstrate that the CAE-DGP networks have better learned to disentangle the markers and the latents, a desirable property for more in-depth behavioral analysis. Furthermore, we find through an unbiased, quantitative assessment of disentangling, that using DGP markers in these models leads to higher levels of disentangling between latents and markers than DLC across many different animal poses present in this dataset (see Figure S1).

\section{Discussion}

In this work, we proposed a probabilistic graphical model built on top of deep neural networks, Deep Graph Pose (DGP), which leverages the rich spatial and temporal structures pervasive in behavioral videos. We also developed an efficient structured variational approach to perform inference in this model. The resulting semi-supervised model exploits information from both labeled and unlabeled frames to achieve significantly more accurate and robust tracking, using fewer labels. Our results illustrate how the smooth behavioral trajectories from DGP lead to improved downstream applications, including the discovery of behavioral "syllables," and interpretable or "disentangled" low-dimensional features from the behavioral videos.

An important direction for future work is to optimize the code to perform online inference for real-time experiments, as in [44]. We are currently integrating DGP on the "Neuroscience Cloud Analysis as a Service" (NeuroCAAS) platform [45], to help enable more scalable and reproducible analyses. Another important direction for future work is to extend our method to operate in 3D, fusing information from multiple cameras. Our variational inference approach should be extensible to this case, using similar epipolar constraints as in $[25,46]$ (using different inference approaches) to perform semi-supervised inference across views. In addition, $[4,5,6]$ all use slightly different architectures and achieve similar accuracies. We plan to perform more experiments with the architectures from $[5,6]$ in the future. Finally, we would like to incorporate our model into existing toolboxes and GUIs to facilitate user access. 


\section{Broader Impact}

We propose a new method for animal behavioral tracking. As highlighted in the introduction and in [10], recent years have seen a rapid increase in the development of methods for animal pose estimation, which need to operate in a different regime than methods developed for human pose estimation. Our work significantly improves the state of the art for animal pose estimation, and thus advances behavioral analysis for animal research, an essential task for scientific discovery in fields ranging from neuroscience to ecology. Finally, our work represents a compelling fusion of deep learning methods with probabilistic graphical model approaches to statistical inference, and we hope to see more fruitful interactions between these rich topic areas in the future.

\section{Acknowledgments and Disclosure of Funding}

We thank the authors of DeepLabCut [4] for generously sharing their code and data. This work was supported by grants from the Wellcome Trust (209558 and 216324) (LP), the Simons Foundation (LP, AN, NM, ES, JC, AW, MW), Gatsby Charitable Foundation GAT3708 (EB, AW, MW), the Searle Scholars Program (AB), Klingenstein-Simons Fellowship (AB), Sloan Foundation Fellowship (AB), Helen Hay Whitney Fellowship (ER), NIH grant NS116734 (AB), NIH Vision Sciences Training Grant EY013933 (CE), NIH T32 (MH015144) (ER), NIH U19NS104649 (Costa U19) (JC), NIH RF1MH120680 (Adesnik) (LP), NIH UF1NS107696 (Ji) (LP), NIH U19NS107613 (Miller U19) (LP, MW, EB, AW), NSF GRFP: DGE 16-44869 (NM), and NSF DBI-1707398 (Neuronex) (LP, JC, MW, $\mathrm{EB}, \mathrm{AW})$. 


\section{References}

[1] Carsen Stringer, Marius Pachitariu, Nicholas Steinmetz, Charu Bai Reddy, Matteo Carandini, and Kenneth D Harris. Spontaneous behaviors drive multidimensional, brainwide activity. Science, 364(6437):eaav7893, 2019.

[2] Nicholas A Steinmetz, Peter Zatka-Haas, Matteo Carandini, and Kenneth D Harris. Distributed coding of choice, action and engagement across the mouse brain. Nature, 576(7786):266-273, 2019.

[3] Simon Musall, Matthew T Kaufman, Ashley L Juavinett, Steven Gluf, and Anne K Churchland. Single-trial neural dynamics are dominated by richly varied movements. Nature neuroscience, 22(10):1677-1686, 2019.

[4] Alexander Mathis, Pranav Mamidanna, Kevin M Cury, Taiga Abe, Venkatesh N Murthy, Mackenzie Weygandt Mathis, and Matthias Bethge. Deeplabcut: markerless pose estimation of user-defined body parts with deep learning. Technical report, Nature Publishing Group, 2018.

[5] Jacob M Graving, Daniel Chae, Hemal Naik, Liang Li, Benjamin Koger, Blair R Costelloe, and Iain D Couzin. Deepposekit, a software toolkit for fast and robust animal pose estimation using deep learning. eLife, 8:e47994, 2019.

[6] Talmo D Pereira, Diego E Aldarondo, Lindsay Willmore, Mikhail Kislin, Samuel S-H Wang, Mala Murthy, and Joshua W Shaevitz. Fast animal pose estimation using deep neural networks. Nature methods, 16(1):117, 2019.

[7] Eldar Insafutdinov, Leonid Pishchulin, Bjoern Andres, Mykhaylo Andriluka, and Bernt Schiele. Deepercut: A deeper, stronger, and faster multi-person pose estimation model. In European Conference on Computer Vision, pages 34-50. Springer, 2016.

[8] Alejandro Newell, Kaiyu Yang, and Jia Deng. Stacked hourglass networks for human pose estimation. In European conference on computer vision, pages 483-499. Springer, 2016.

[9] Qi Dang, Jianqin Yin, Bin Wang, and Wenqing Zheng. Deep learning based 2d human pose estimation: A survey. Tsinghua Science and Technology, 24(6):663-676, 2019.

[10] Mackenzie Weygandt Mathis and Alexander Mathis. Deep learning tools for the measurement of animal behavior in neuroscience. Current Opinion in Neurobiology, 60:1-11, 2020.

[11] Virginie Uhlmann, Pavan Ramdya, Ricard Delgado-Gonzalo, Richard Benton, and Michael Unser. Flylimbtracker: An active contour based approach for leg segment tracking in unmarked, freely behaving drosophila. PLoS One, 12(4), 2017.

[12] Praneet C Bala, Benjamin R Eisenreich, Seng Bum Michael Yoo, Benjamin Y Hayden, Hyun Soo Park, and Jan Zimmermann. Openmonkeystudio: Automated markerless pose estimation in freely moving macaques. bioRxiv, 2020.

[13] Oliver Sturman, Lukas Matthias von Ziegler, Christa Schälppi, Furkan Akyol, Benjamin Friedrich Grewe, and Johannes Bohacek. Deep learning based behavioral analysis enables high precision rodent tracking and is capable of outperforming commercial solutions. bioRxiv, 2020.

[14] XiaoLe Liu, Si-yang Yu, Nico Flierman, Sebastian Loyola, Maarten Kamermans, Tycho M Hoogland, and Chris I De Zeeuw. Optiflex: video-based animal pose estimation using deep learning enhanced by optical flow. BioRxiv, 2020.

[15] Xianjie Chen and Alan L Yuille. Articulated pose estimation by a graphical model with image dependent pairwise relations. In Advances in neural information processing systems, pages 1736-1744, 2014.

[16] Guoqiang Liang, Xuguang Lan, Jiang Wang, Jianji Wang, and Nanning Zheng. A limb-based graphical model for human pose estimation. IEEE Transactions on Systems, Man, and Cybernetics: Systems, 48(7):1080-1092, 2017.

[17] Steven Schwarcz and Thomas Pollard. 3d human pose estimation from deep multi-view 2d pose. In 2018 24th International Conference on Pattern Recognition (ICPR), pages 2326-2331. IEEE, 2018.

[18] Deying Kong, Yifei Chen, Haoyu Ma, Xiangyi Yan, and Xiaohui Xie. Adaptive graphical model network for 2d handpose estimation. arXiv preprint arXiv:1909.08205, 2019.

[19] Jie Song, Limin Wang, Luc Van Gool, and Otmar Hilliges. Thin-slicing network: A deep structured model for pose estimation in videos. In Proceedings of the IEEE conference on computer vision and pattern recognition, pages 4220-4229, 2017. 
[20] Gedas Bertasius, Christoph Feichtenhofer, Du Tran, Jianbo Shi, and Lorenzo Torresani. Learning temporal pose estimation from sparsely-labeled videos. In Advances in Neural Information Processing Systems, pages 3027-3038, 2019.

[21] Xiaojin Zhu, Zoubin Ghahramani, and John D Lafferty. Semi-supervised learning using gaussian fields and harmonic functions. In Proceedings of the 20th International conference on Machine learning (ICML-03), pages 912-919, 2003.

[22] O. Chapelle, B. Schölkopf, and A. Zien. Semi-supervised Learning. Adaptive computation and machine learning. MIT Press, 2010.

[23] Alexander Ratner, Stephen H Bach, Henry Ehrenberg, Jason Fries, Sen Wu, and Christopher Ré. Snorkel: Rapid training data creation with weak supervision. The VLDB Journal, pages 1-22, 2019.

[24] Norimichi Ukita and Yusuke Uematsu. Semi-and weakly-supervised human pose estimation. Computer Vision and Image Understanding, 170:67-78, 2018.

[25] Yilun Zhang and Hyun Soo Park. Multiview supervision by registration. arXiv preprint arXiv:1811.11251, 2018.

[26] Russell Stewart and Stefano Ermon. Label-free supervision of neural networks with physics and domain knowledge. In Thirty-First AAAI Conference on Artificial Intelligence, 2017.

[27] Alexander Mordvintsev and Abid Rahman K. Optical Flow in OpenCV, 2013. https: //opencv-python-tutroals.readthedocs.io/en/latest/py_tutorials/py_video/py_ lucas_kanade/py_lucas_kanade.html.

[28] David M Blei, Alp Kucukelbir, and Jon D McAuliffe. Variational inference: A review for statisticians. Journal of the American Statistical Association, 112(518):859-877, 2017.

[29] Wu Lin, Nicolas Hubacher, and Mohammad Emtiyaz Khan. Variational message passing with structured inference networks. arXiv preprint arXiv:1803.05589, 2018.

[30] G. T. Meijer, M. M. Schartner, V. Aguillon, N. Bonacchi, M. Carandini, F. Cazettes, G. A. Chapius, A. K. Churchland, Y. Dan, E. E. J. Dewitt, H. Martinez Vergara, M. Faulkner, M. Hausser, F. Hu, I. C. Laranjeira, Z. F. Mainen, N. J. Miska, T. D. Mrsic-flogel, J. P. Noel, A. Pan Vazquez, L. M. Paninski, A. Pouget, K. Z. Socha, K. Svoboda, A. E. Urai, M. R. Whiteway, O. Winter, and IBL Collaboration. Robust and generalizable tracking of body parts of head-fixed mice. In SFN, 2019.

[31] Mackenzie Weygandt Mathis, Alexander Mathis, and Naoshige Uchida. Somatosensory cortex plays an essential role in forelimb motor adaptation in mice. Neuron, 93(6):1493-1503, 2017.

[32] Evan Schaffer, Neeli Mishra, Wenze Li, Matthew Whiteway, Jason Freedman, Kripa Patel, Venkatakaushik Voleti, Liam Paninski, Larry Abbott, Elizabeth Hillman, and Richard Axel. Flygenvectors: large-scale dynamics of internal and behavioral states in a small animal. In Cosyne, 2020.

[33] Amy L. Norovich*, Claire P. Everett*, Taiga Abe, and Andrés Bendesky. Probing the neural basis of visually-evoked aggression in siamese fighting fish. In Cold Spring Harbor Zebrafish Neural Circuits and Behavior, November 20-23, Cold Spring Harbor, NY, USA, 2019.

[34] The International Brain Laboratory, Valeria Aguillon-Rodriguez, Dora E. Angelaki, Hannah M. Bayer, Niccolò Bonacchi, Matteo Carandini, Fanny Cazettes, Gaelle A. Chapuis, Anne K. Churchland, Yang Dan, Eric E. Dewitt, Mayo Faulkner, Hamish Forrest, Laura M. Haetzel, Michael Hausser, Sonja B. Hofer, Fei Hu, Anup Khanal, Christopher S. Krasniak, Inês Laranjeira, Zachary F. Mainen, Guido T. Meijer, Nathaniel J. Miska, Thomas D. Mrsic-Flogel, Masayoshi Murakami, Jean-Paul Noel, Alejandro Pan-Vazquez, Josh I. Sanders, Karolina Z. Socha, Rebecca Terry, Anne E. Urai, Hernando M. Vergara, Miles J. Wells, Christian J. Wilson, Ilana B. Witten, Lauren E. Wool, and Anthony Zador. A standardized and reproducible method to measure decision-making in mice. bioRxiv, 2020.

[35] Alexander B Wiltschko, Matthew J Johnson, Giuliano Iurilli, Ralph E Peterson, Jesse M Katon, Stan L Pashkovski, Victoria E Abraira, Ryan P Adams, and Sandeep Robert Datta. Mapping sub-second structure in mouse behavior. Neuron, 88(6):1121-1135, 2015.

[36] Matthew J Johnson, David K Duvenaud, Alex Wiltschko, Ryan P Adams, and Sandeep R Datta. Composing graphical models with neural networks for structured representations and fast inference. In Advances in neural information processing systems, pages 2946-2954, 2016. 
[37] Eleanor Batty, Matthew Whiteway, Shreya Saxena, Dan Biderman, Taiga Abe, Simon Musall, Winthrop Gillis, Jeffrey Markowitz, Anne Churchland, John P Cunningham, et al. Behavenet: nonlinear embedding and bayesian neural decoding of behavioral videos. In Advances in Neural Information Processing Systems, pages 15680-15691, 2019.

[38] Irina Higgins, Loic Matthey, Arka Pal, Christopher Burgess, Xavier Glorot, Matthew Botvinick, Shakir Mohamed, and Alexander Lerchner. beta-vae: Learning basic visual concepts with a constrained variational framework. Iclr, 2(5):6, 2017.

[39] Tian Qi Chen, Xuechen Li, Roger B Grosse, and David K Duvenaud. Isolating sources of disentanglement in variational autoencoders. In Advances in Neural Information Processing Systems, pages 2610-2620, 2018.

[40] Antonia Creswell, Anil A Bharath, and Biswa Sengupta. Conditional autoencoders with adversarial information factorization. arXiv preprint arXiv:1711.05175, 2017.

[41] Guillaume Lample, Neil Zeghidour, Nicolas Usunier, Antoine Bordes, Ludovic Denoyer, and Marc'Aurelio Ranzato. Fader networks: Manipulating images by sliding attributes. In Advances in Neural Information Processing Systems, pages 5967-5976, 2017.

[42] Jack Klys, Jake Snell, and Richard Zemel. Learning latent subspaces in variational autoencoders. In Advances in Neural Information Processing Systems, pages 6444-6454, 2018.

[43] Xiao Li, Chenghua Lin, Chaozheng Wang, and Frank Guerin. Latent space factorisation and manipulation via matrix subspace projection. arXiv preprint arXiv:1907.12385, 2019.

[44] Jens F Schweihoff, Matvey Loshakov, Irina Pavlova, Laura Kück, Laura A Ewell, and Martin K Schwarz. Deeplabstream: Closing the loop using deep learning-based markerless, real-time posture detec-tion. bioRxiv, 2019.

[45] Taiga Abe, Ian Kinsella, Shreya Saxena, Liam Paninski, and John P Cunningham. Neuroscience cloud analysis as a service. bioRxiv, 2020.

[46] Semih Günel, Helge Rhodin, Daniel Morales, João Campagnolo, Pavan Ramdya, and Pascal Fua. Deepfly3d, a deep learning-based approach for $3 \mathrm{~d}$ limb and appendage tracking in tethered, adult drosophila. eLife, 8, 2019.

[47] James M Varah. On the solution of block-tridiagonal systems arising from certain finite-difference equations. Mathematics of Computation, 26(120):859-868, 1972.

[48] Luca Guido Molinari. Determinants of block tridiagonal matrices. Linear algebra and its applications, 429(8-9):2221-2226, 2008.

[49] Matthew G Reuter and Judith C Hill. An efficient, block-by-block algorithm for inverting a block tridiagonal, nearly block toeplitz matrix. Computational Science \& Discovery, 5(1):014009, 2012.

[50] Diederik P Kingma and Jimmy Ba. Adam: A method for stochastic optimization. arXiv preprint arXiv:1412.6980, 2014.

[51] Gregory Druck, Burr Settles, and Andrew McCallum. Active learning by labeling features. In Proceedings of the 2009 conference on Empirical methods in natural language processing, pages 81-90, 2009.

[52] Burr Settles. From theories to queries: Active learning in practice. In Active Learning and Experimental Design workshop In conjunction with AISTATS 2010, pages 1-18, 2011.

[53] Burr Settles. Active learning literature survey. Technical report, University of Wisconsin-Madison Department of Computer Sciences, 2009.

[54] Hamed H Aghdam, Abel Gonzalez-Garcia, Joost van de Weijer, and Antonio M López. Active learning for deep detection neural networks. In Proceedings of the IEEE International Conference on Computer Vision, pages 3672-3680, 2019. 


\section{S1 Expanded methods}

In this section we present our model and inference approach in fuller detail than was possible given space limitations in the main text. (To maintain the logical flow, in some cases we repeat points that were made in the main text methods.)

\section{S1.1 Deep Graph Pose model}

The graphical model of DGP is summarized in Figure 1 . We observe frames $x_{t}$ indexed by $t$, along with a small subset of labeled markers $y_{t, j}$ (where $j$ indexes the different targets we would like to track). The target locations $y_{t, j}$ on most frames are unlabeled, but we have several sources of information to constrain these latent variables: temporal smoothness constraints between the targets $y_{t, j}$ and $y_{t+1, j}$, which we capture with quadratic potentials $\phi_{t}$; spatial constraints between the targets $y_{t, i}$ and $y_{t, j}$, modeled with quadratic potentials $\phi_{s}$; and information from the image $x_{t}$, modeled by potentials $\phi_{n}$ parametrized by neural networks.

First let's define the potential function $\phi_{n}$ between the input image $x$ and the target's 2D location $y$. We define $f_{\theta}(\cdot)$ as a stack of a fixed pretrained ResNet-50 network and a trainable ConvNet parametrized by $\theta . f_{\theta}(\cdot)$ takes a frame $x$ as the input and outputs a 2D affinity map image, which ideally has a sharp peak at the most likely coordinates of the target. We then denote the sigmoid function as $\sigma(\cdot)$, and refer to $\sigma\left(f_{\theta}(x)\right)$ as a "confidence map."

With the potential $\phi_{n}$, our target is to match this 2D confidence map to a 2D Gaussian bump centered at $y$ by minimizing the sigmoid cross entropy. Now let's define the Gaussian bump. We construct a bivariate Gaussian function with mean $y=\left[y_{m}, y_{n}\right]$ and variance $l^{2}$. The Gaussian function at the $m$ th row and the $n$th column is

$$
G\left(y, l^{2}\right)_{m n}=\frac{1}{2 \pi l^{2}} \exp \left(-\frac{1}{2 l^{2}}\left(m-y_{m}\right)^{2}-\frac{1}{2 l^{2}}\left(n-y_{n}\right)^{2}\right)
$$

The variance parameter was set as $l^{2}=1$ in practice.

The potential function $\phi_{n}$ for the $m$ th row and the $n$th column entry in $\sigma\left(f_{\theta}(x)\right)$ is defined as

$$
\begin{aligned}
\phi_{n}(y, x)_{m n} & =\frac{1}{2} w_{n}\left(-G\left(y, l^{2}\right)_{m n} \cdot \log \left(\sigma\left(f_{\theta}(x)\right)_{m n}\right)-\left(1-G\left(y, l^{2}\right)_{m n}\right) \cdot \log \left(1-\sigma\left(f_{\theta}(x)\right)_{m n}\right)\right) \\
& =\frac{1}{2} w_{n}\left(-f_{\theta}(x)_{m n} \cdot G\left(y, l^{2}\right)_{m n}+f_{\theta}(x)_{m n}+\log \left(1+\exp \left(-f_{\theta}(x)_{m n}\right)\right)\right) .
\end{aligned}
$$

Summing over all entries in the confidence map, we get the neural network potential $\phi_{n}$ as

$$
\phi_{n}(y, x)=\sum_{m, n} \frac{1}{2} w_{n}\left(-f_{\theta}(x)_{m n} \cdot G\left(y, l^{2}\right)_{m n}+f_{\theta}(x)_{m n}+\log \left(1+\exp \left(-f_{\theta}(x)_{m n}\right)\right)\right) .
$$

We will write everything in vector form hereafter. We define $\mathbf{f}$ as the vectorized $f_{\theta}(x)$, define $\mathbf{h}$ as the vectorized $f_{\theta}(x)+\log \left(1+\exp \left(-f_{\theta}(x)\right)\right)$, and define $\mathbf{G}\left(y, l^{2}\right)$ as the vectorized $G\left(y, l^{2}\right)$, which is a function of mean $y$ and variance $l^{2}$. Thus, for each target $j$ we can rewrite the $j$-th image-based potential $\phi_{n}^{j}$ as

$$
\phi_{n}^{j}\left(y_{t, j}, x_{t}\right)=\frac{1}{2} w_{n}\left(-\mathbf{f}_{t, j}^{\top} \mathbf{G}\left(y_{t, j}, l^{2}\right)+\mathbf{h}_{t, j}^{\top} \mathbf{1}\right),
$$

where $j$ is the index for target $j$ and $t$ is the index for frame $t$.

We use a simple quadratic potential $\phi_{t}$ to impose temporal smoothness:

$$
\phi_{t}^{j}\left(y_{t, j}, y_{t+1, j}\right)=\frac{1}{2} w_{t}^{j}\left\|y_{t, j}-y_{t+1, j}\right\|^{2},
$$

which penalizes the distance between targets in consecutive frames; the weights $w_{t}^{j}$ in general may depend on the target index $j$, and can also vary in time. A more sophisticated version of the temporal clique could be an $L_{2}$ norm over the second or third order temporal difference, similar to optical flow.

The spatial potential $\phi_{s}$ is more dataset-dependent and can be chosen depending on the constraints that the markers should satisfy. Typical examples include a soft constraint that the paw marker should not exceed some distance from the elbow marker, or the nose should always stay within a certain radius of a static waterspout. This can be achieved by a soft-thresholding quadratic loss, leading to 
a smooth pairwise potential between these two markers. Again, we start with a simple quadratic potential to encode these soft constraints:

$$
\phi_{s}^{i j}\left(y_{t, i}, y_{t, j}\right)=\frac{1}{2} w_{s}^{i j}\left\|y_{t, i}-y_{t, j}\right\|^{2},
$$

which penalizes the distance between "connected" targets $y_{t, i}$ and $y_{t, j}$ (where the user can pre-specify pairs of connected targets that should have neighboring locations in the frame, e.g. paw and elbow).

We want to "let the data speak" and avoid oversmoothing, so the penalty weights $w_{s}$ and $w_{t}$ should be small. In practice we found that the temporal weights $w_{t}^{j}$ could be set using optical flow [27] which captures the vector field between neighbor frames. We first computed the vector field between two neighbor frames $t-1$ and $t$ using optical flow. Then we calculated the average motion vector for target $j$ from frame $t-1$ to frame $t$. The magnitude of the motion vector was denoted as $m_{t}^{j}$. Finally $w_{t}^{j}=\xi / m_{t}^{j}$, where $\xi$ is a constant scalar independent of dataset, time and target indices. The intuition is the larger the movement of the target is, the smaller the temporal clique weight should be. We set the spatial weights as $w_{s}^{i j}=c / d_{i j}$, where $d_{i j}$ is a rough estimate of the average distance (in pixels) between targets $i$ and $j$ and $c>0$ is a small scalar (again independent of dataset and target indices $i, j$ ), led to robust results without any need to fit extra parameters.

We summarize the parameter vector as $\beta=\left\{\theta, w_{n}, w_{t}, w_{s}\right\}$, where $\theta$ denotes the neural net parameters. Given $\beta$ and the full collection of images $x$, the joint probability distribution over targets $y$ is

$$
p(y \mid x, \beta)=\frac{1}{Z(x, \beta)} \exp (\underbrace{-\sum_{t=1}^{T} \sum_{j=1}^{J} \phi_{n}^{j}\left(y_{t, j}, x_{t}\right.}_{\text {neural network }})-\underbrace{\sum_{t=1}^{T-1} \sum_{j=1}^{J} \phi_{t}^{j}\left(y_{t, j}, y_{t+1, j}\right)-\sum_{t=1}^{T} \sum_{i, j \in \mathcal{E}} \phi_{s}^{i j}\left(y_{t, i}, y_{t, j}\right)}_{\text {Gaussian graphical model }})
$$

where $\mathcal{E}$ denotes the edge set of constrained targets (i.e., the pairs $i, j$ with a nonzero potential function), $Z(x, \beta)=\int p(y \mid x, \beta) d y$ is the normalizing constant marginalizing out $y, T$ denotes the total number of frames, and $J$ denotes the total number of targets. The joint distribution can be described as a combination of a neural network component and a probabilistic graphical model over the latent variables (the unobserved targets $y$ ).

\section{S1.2 Structured variational inference}

Our goal is to estimate $p\left(y^{h} \mid y^{v}, x, \beta\right)$, the posterior over locations of unlabeled targets $y^{h}$, given the frames from the video $x$, the locations of the labeled markers $y^{v}$, and the parameters $\beta$. (Here $h$ denotes hidden, for the unlabeled data, and $v$ denotes visible, for the labeled data.) Calculating this posterior distribution exactly is intractable, due to the highly nonlinear potentials $\phi_{n}$. We chose to use structured variational inference, similar to [29], to approximate this posterior. We approximate $p\left(y^{h}, y^{v} \mid x, \beta\right)$ with a Gaussian graphical model (GGM) with the same graphical model as Figure 1. We denote the approximate posterior as $q\left(y^{h}, y^{v} \mid x, \beta_{q}\right)$ ( $\beta_{q}$ encodes variational parameters). To obtain a fully Gaussian variational approximation, we replace the neural network potentials $\phi_{n}^{j}$ with quadratic terms

$$
\hat{\phi}_{n}^{j}\left(y_{t, j}, x_{t}\right)=\frac{1}{2} w_{n, q}^{t, j}\left\|y_{t, j}-\mu_{n}^{t, j}\left(x_{t}\right)\right\|^{2} .
$$

Here the precision variables $w_{n, q}^{t, j}$ and means $\mu_{n}^{t, j}$ are variational parameters that we could optimize over independently. However, we found it more efficient to model the means $\mu_{n}$ as $\mu_{n}^{t, j}\left(x_{t}\right)=$ $\sum_{m, n} \alpha_{m n} \operatorname{Softmax}\left(f_{\gamma}^{j}\left(x_{t}\right)\right)_{m n}$, where $\alpha_{m n}=[m, n]$. Here $f_{\gamma}(\cdot)$ is an inference neural network with parameters $\gamma$ whose output is a 2D affinity map, similar to $f_{\theta}(\cdot)$. Putting the pieces together, we have the fully Gaussian approximate posterior

$$
q\left(y^{h}, y^{v} \mid x, \beta_{q}\right)=\frac{1}{\hat{Z}\left(x, \beta_{q}\right)} \exp (\underbrace{-\sum_{t=1}^{T} \sum_{j=1}^{J} \hat{\phi}_{n}^{j}\left(y_{t, j}, x_{t}\right)}_{\text {inference network }}-\underbrace{\sum_{t=1}^{T-1} \sum_{j=1}^{J} \phi_{t}^{j}\left(y_{t, j}, y_{t+1, j}\right)-\sum_{t=1}^{T} \sum_{i, j \in \mathcal{E}} \phi_{s}^{i j}\left(y_{t, i}, y_{t, j}\right)}_{\text {identical to equation } \mathrm{S} 8}),
$$

where $\hat{Z}\left(x, \beta_{q}\right)$ is the normalizing constant (which can be computed explicitly, due to the fullyGaussian form of $q$ ), and $\beta_{q}=\left\{\gamma, w_{n, q}, w_{t}, w_{s}\right\}$. 
Since $q\left(y^{h}, y^{v} \mid x, \beta_{q}\right)$ is a GGM, we can rewrite eq. S10 in the standard Gaussian form

$$
\begin{aligned}
q\left(y^{h}, y^{v} \mid x, \beta_{q}\right) & =\mathcal{N}\left(\mu_{a}, \Sigma_{a}\right) \\
\Sigma_{a} & =\left(\Sigma_{s}^{-1}+\Sigma_{t}^{-1}+\Sigma_{n}^{-1}\right)^{-1} \\
\mu_{a} & =\Sigma_{a} \Sigma_{n}^{-1} \mu_{n}
\end{aligned}
$$

where $\Sigma_{n}^{-1}, \Sigma_{t}^{-1}$ and $\Sigma_{s}^{-1}$ are the precision matrices corresponding to the potentials in $q\left(y^{h}, y^{v} \mid x, \beta_{q}\right)$; these have the form

$$
\begin{aligned}
& \Sigma_{n}^{-1}=\left[\begin{array}{cccccc}
w_{n, q}^{1,1} & 0 & 0 & 0 & 0 & 0 \\
0 & w_{n, q}^{1,2} & 0 & 0 & 0 & 0 \\
0 & 0 & w_{n, q}^{2,1} & 0 & 0 & 0 \\
0 & 0 & 0 & w_{n, q}^{2,2} & 0 & 0 \\
0 & 0 & 0 & 0 & w_{n, q}^{3,1} & 0 \\
0 & 0 & 0 & 0 & 0 & w_{n, q}^{3,2}
\end{array}\right] \\
& \Sigma_{t}^{-1}=\left[\begin{array}{cccccc}
w_{t}^{1} & 0 & -w_{t}^{1} & 0 & 0 & 0 \\
0 & w_{t}^{2} & 0 & -w_{t}^{2} & 0 & 0 \\
-w_{t}^{1} & 0 & 2 w_{t}^{1} & 0 & -w_{t}^{1} & 0 \\
0 & -w_{t}^{2} & 0 & 2 w_{t}^{2} & 0 & -w_{t}^{2} \\
0 & 0 & -w_{t}^{1} & 0 & w_{t}^{1} & 0 \\
0 & 0 & 0 & -w_{t}^{2} & 0 & w_{t}^{2}
\end{array}\right] \\
& \Sigma_{s}^{-1}=\left[\begin{array}{cccccc}
w_{s} & -w_{s} & 0 & 0 & 0 & 0 \\
-w_{s} & w_{s} & 0 & 0 & 0 & 0 \\
0 & 0 & w_{s} & -w_{s} & 0 & 0 \\
0 & 0 & -w_{s} & w_{s} & 0 & 0 \\
0 & 0 & 0 & 0 & w_{s} & -w_{s} \\
0 & 0 & 0 & 0 & -w_{s} & w_{s}
\end{array}\right]
\end{aligned}
$$

Thus the mean and covariance of the variational distribution $q\left(y^{h}, y^{v} \mid x, \beta_{q}\right)$ are $\mu_{a}$ and $\Sigma_{a}$, where $\mu_{a}$ is a function of $\gamma, w_{n, q}, w_{t}$, and $w_{s}$, and $\Sigma_{a}$ is a function of $w_{n, q}, w_{t}$, and $w_{s}$.

Let $P_{h}$ and $P_{v}$ denote the permutation matrices that map the vector $y$ to $y^{h}$ and $y^{v}$ respectively, i.e.,

$$
y^{h}=P_{h} y, \quad y^{v}=P_{v} y
$$

Due to the Gaussianity of the joint distribution, we can write down the closed-form expression for $q\left(y^{h} \mid y^{v}, x, \beta_{q}\right)$ as

$$
q\left(y^{h} \mid y^{v}, x, \beta_{q}\right)=\mathcal{N}\left(\mu_{h}, \Sigma_{h}\right)
$$

where

$$
\begin{aligned}
\Sigma_{h} & =\left(P_{h} \Sigma_{a}^{-1} P_{h}^{\top}\right)^{-1}, \\
\mu_{h} & =P_{h} \mu_{a}-\left(P_{h} \Sigma_{a}^{-1} P_{h}^{\top}\right)^{-1} P_{h} \Sigma_{a}^{-1} P_{v}^{\top}\left(y^{v}-P_{v} \mu_{a}\right) .
\end{aligned}
$$




\section{S1.2.1 Evidence Lower Bound (ELBO)}

Given the approximate posterior (eq. S17), and abbreviating $q\left(y^{h}\right)=q\left(y^{h} \mid y^{v}, x, \beta_{q}\right)$, we can now write down the evidence lower bound (ELBO) as

$$
\begin{aligned}
\mathcal{L}= & \mathbb{E}_{q\left(y^{h}\right)}\left[-\log q\left(y^{h}\right)+\log p\left(y^{h}, y^{v} \mid x, \beta\right)\right] \\
= & \mathbb{E}_{q\left(y^{h}\right)}\left[\log p\left(y^{h}, y^{v} \mid x, \beta\right)\right]+H(q) \\
= & -\mathbb{E}_{q\left(y^{h}\right)}\left[\sum_{t=1}^{T} \sum_{j=1}^{J} \phi_{n}^{j}\left(y_{t, j}, x_{t}\right)\right]-\mathbb{E}_{q\left(y^{h}\right)}\left[\sum_{t=1}^{T-1} \sum_{j=1}^{J} \phi_{t}^{j}\left(y_{t, j}, y_{t+1, j}\right)\right]-\mathbb{E}_{q\left(y^{h}\right)}\left[\sum_{t=1}^{T} \sum_{i, j \in \mathcal{E}} \phi_{s}^{i j}\left(y_{t, i}, y_{t, j}\right)\right] \\
& -\log Z(x, \beta)+H(q) \\
= & \sum_{t=1, j=1}^{T, J} \frac{w_{n}}{2}\left(-\mathbf{f}_{t, j}^{\top} \mathbf{G}\left(y_{t}, l^{2}\right)+\mathbf{h}_{t, j}^{\top} \mathbf{1}\right)+\sum_{\substack{t=1, j=1 \\
t, j \in \mathcal{V}}}^{T, J} \frac{w_{n}}{2}\left(-\mathbf{f}_{t, j}^{\top} \mathbf{G}\left(\mu_{h t}, l^{2}+\Sigma_{h t t}\right)+\mathbf{h}_{t, j}^{\top} \mathbf{1}\right) \\
& -\operatorname{Tr}\left(\left(\Sigma_{s}^{-1}+\Sigma_{t}^{-1}\right) P_{h}^{\top} \Sigma_{h} P_{h}\right)-\frac{1}{2} \operatorname{Tr}\left(\left(P_{h}^{\top} \mu_{h}+P_{v}^{\top} y^{v}\right)^{\top}\left(\Sigma_{s}^{-1}+\Sigma_{t}^{-1}\right)\left(P_{h}^{\top} \mu_{h}+P_{v}^{\top} y^{v}\right)\right) \\
& -\log Z(x, \beta)+\log \left|\Sigma_{a}\right|-\log \left|P_{v} \Sigma_{a} P_{v}^{\top}\right|,
\end{aligned}
$$

where $\mathcal{V}$ and $\mathcal{H}$ denote the sets of visible targets in visible frames and hidden targets in all frames respectively.

The bottlenecks of the ELBO computation in the full DGP model are $\Sigma_{a} \Sigma_{n}^{-1} \mu_{n}, \log \left|\Sigma_{a}\right|$, and $\operatorname{diag}\left(\Sigma_{a}\right)$, where $\Sigma_{a} \in \mathbb{R}^{T J \times T J}$ and $\Sigma_{a}^{-1}$ is a block tridiagonal matrix. All of these terms can be computed via message passing with $O\left(T J^{3}\right)$ time complexity, due to the chain structure of the graphical model (and the corresponding block tridiagonal structure of the precision matrix). We used standard message passing algorithms to handle the required block tridiagonal matrix computations $[47,48,49]$.

\section{S1.2.2 Semi-supervised DLC}

To understand the various terms in the ELBO above it is helpful to start with a simpler special case. If we turn off the temporal and spatial potentials in eq. S20 (i.e., set $w_{t}=w_{s}=0$ ) we arrive at the DGP-semi model discussed in the Results section. The corresponding ELBO is

$$
\begin{aligned}
\mathcal{L} & =\sum_{\substack{t=1, j=1 \\
t, j \in \mathcal{V}}}^{T, J} \frac{w_{n}}{2}\left(-\mathbf{f}_{t}^{\top} \mathbf{G}\left(y_{t}, l^{2}\right)+\mathbf{h}_{t}^{\top} \mathbf{1}\right)+\sum_{\substack{t=1, j=1 \\
t, j \in \mathcal{H}}}^{T, J} \frac{w_{n}}{2}\left(-\mathbf{f}_{t}^{\top} \mathbf{G}\left(\mu_{h t}, l^{2}+\Sigma_{h t t}\right)+\mathbf{h}_{t}^{\top} \mathbf{1}\right) \\
& -\log Z(x, \beta)+\log \left|\Sigma_{n}\right|-\log \left|P_{v} \Sigma_{n} P_{v}^{\top}\right|,
\end{aligned}
$$

where $\Sigma_{h}=\left(P_{h} \Sigma_{n} P_{h}^{\top}\right)^{-1}$ and $\mu_{h}=P_{h} \mu_{n}$. The first term is a conventional DLC-type cross entropy for labeled frames. The second term is a semi-supervised cross entropy for unlabeled frames. Instead of having the true marker locations for unobserved frames, we construct the Gaussian function using the $2 \mathrm{D}$ location output from the neural net. The second term encourages the confidence map $f_{\theta}$ to be unimodal to match the Gaussian approximate posterior. This semi-supervised term leads to better performance of DGP-semi compared to the original fully-supervised DLC (Figure 3).

\section{S1.3 Implementation details}

There are a few issues regarding the optimization of the full DGP model that we considered during the implementation:

- In eq. S20, the $\log$ normalization term $\log Z(x, \beta)$ involves an integration over all frames and markers which makes the optimization intractable. In eq. S21, the graphical model factorizes over markers $j$ and frames $t$, which means that we can calculate the $\log$ normalization term $\log Z(x, \beta)$ directly by summing over pixels for each $t$ and $j$. But the summation over all pixels consumes a lot of time. In practice, we found that dropping the $\log Z$ term did not affect the results significantly. 
- The optimization of the full ELBO involves two steps - expectation (E) and maximization (M). We estimate a good $q$ distribution in the E-step and optimize the network parameters given the $q$ distribution in the M-step. However, we found that in practice the E-step for the full DGP model was very time-consuming and only marginally improved the performance. Thus during the implementation, we simplified the $q$ distribution by dropping the temporal and spatial cliques in eq. S10, but still kept the full graph for $p$ as in eq. S8. Equivalently, this simplified the ELBO as well. The $q$ distribution optimized in the E-step can now factorize over frames thus making the computation a lot faster.

- With the simplified ELBO, the unknown parameters are $\left\{\theta, \gamma, w_{n}, w_{t}, w_{s}, w_{n, q}\right\} . \quad \gamma$ and $w_{n, q}$ are the unknown parameters we need to learn during the E-step. We found that setting $\gamma=\theta$ led to good results and reduced the number of parameters. Moreover, optimizing $w_{n, q}$ didn't significantly improve the performance. Thus, for computational considerations, we decided to skip the E-step and set $w_{n, q}=1$, which is a reasonable precision for the Gaussian bump, and set $\gamma=\theta$. In the M-step, we fixed $w_{t}^{j}$ using optical flow which provided the vector field of the dynamics between two neighbor frames, as described earlier. We set $w_{s}^{i j}=c / d_{i j}$, where $d_{i j}$ is the average distance (in pixels) between targets $i$ and $j$ and $c>0$ is a small scalar (independent of dataset and target indices $i, j$ ); this led to robust results without any need to fit extra parameters. We also differentiated $w_{n}$ to be $w_{n}^{v}$ and $w_{n}^{h}$ for visible and hidden frames. Empirically, $w_{n}^{h}=3$ and $w_{n}^{v}=2 w_{n}^{h} T / T_{v}\left(T_{v}\right.$ is the number of visible frames) led to good results; this upweighted the strength of labeled frames relative to unobserved frames. Therefore, the only parameter left is $\theta$.

- When simplifying the objective function, we can get rid of the harsh contraints on the form of the temporal and spatial cliques. The reason we choose both to be $L_{2}$ norms as in eq. S6 and S7 is that only $L_{2}$ norms in the $q$ distribution can lead to a closed-form expectation in the ELBO. However, if we don't consider these two cliques in the $q$ distribution, we can allow arbitrary forms. In the experiment, we still employed eq. S6 for the temporal clique, but employed a soft-thresholding quadratic loss $\phi_{s}^{i j}\left(y_{t, i}, y_{t, j}\right)=\frac{1}{2} w_{s}^{i j} \operatorname{Relu}\left[\left\|y_{t, i}-y_{t, j}\right\|^{2}-d_{i j}\right]$ for the spatial clique, where $d_{i j}$ is the average distance (in pixels) between targets $i$ and $j$. This spatial clique penalizes two markers when their distance is above the average distance calculated from the ground truth labels.

Therefore, the final objective function is

$$
\begin{aligned}
\mathcal{L}(\theta)= & \sum_{\substack{t=1, j=1 \\
t, j \in \mathcal{V}}}^{T, J} \frac{w_{n}}{2}\left(-\mathbf{f}_{t, j}^{\top} \mathbf{G}\left(y_{t}, l^{2}\right)+\mathbf{h}_{t, j}^{\top} \mathbf{1}\right)+\sum_{\substack{t=1, j=1 \\
t, j \in \mathcal{H}}}^{T, J} \frac{w_{n}}{2}\left(-\mathbf{f}_{t, j}^{\top} \mathbf{G}\left(\mu_{h t}, l^{2}+\Sigma_{h t t}\right)+\mathbf{h}_{t, j}^{\top} \mathbf{1}\right) \\
& -\frac{1}{2} \sum_{t=1}^{T-1} \sum_{j=1}^{J} w_{t}^{j}\left\|\mu_{t, j}-\mu_{t+1, j}\right\|^{2}-\sum_{t=1}^{T} \sum_{i, j \in \mathcal{E}} w_{s}^{i j} \operatorname{Relu}\left[\left\|\mu_{t, i}-\mu_{t, j}\right\|^{2}-d_{i j}\right], \quad \text { (S22) }
\end{aligned}
$$

where $\mu_{t, j}=y_{t, j}$ if $(t, j)$ is labeled; $\mu_{t, j}=\mu_{h t, j}$ otherwise. We maximized the above objective function and calculated the gradients for $\theta$ using standard automatic differentiation tools, and performed standard stochastic gradient updates to estimate these parameters.

\section{S2 Conditional convolutional autoencoder}

\section{S2.1 Implementation details}

We fit conditional convolutional autoencoders (conditional CAEs) on 192x192 grayscale images from [30]. In addition, we used 4 markers output by DLC/DGP: left paw, right paw, tongue, and nose. To condition the encoder network on these values we turned each marker into a one-hot 2D array and concatenated these with the corresponding frame, so that the input to the encoder was of size $(192,192,5)$. To condition the decoder network on these values we first centered the marker values by subtracting their median (computed over the entire dataset) and then concatenated these values to the latents before feeding them into the decoder. See Table S1 for network architecture details. We trained the autoencoders by minimizing the MSE between original and reconstructed frames using the Adam optimizer [50] with a learning rate of $10^{-4}$, a batch size of 100 , and no regularization. Models were trained for 300 epochs. 


\begin{tabular}{|c|c|c|c|c|c|c|}
\hline Layer & Type & Channels & Kernel Size & Stride Size & Zero Padding & Output Size \\
\hline \hline 0 & conv & 32 & $(5,5)$ & $(2,2)$ & $(1,2,1,2)$ & $(96,96,32)$ \\
\hline 1 & conv & 64 & $(5,5)$ & $(2,2)$ & $(1,2,1,2)$ & $(48,48,64)$ \\
\hline 2 & conv & 128 & $(5,5)$ & $(2,2)$ & $(1,2,1,2)$ & $(24,24,128)$ \\
\hline 3 & conv & 256 & $(5,5)$ & $(2,2)$ & $(1,2,1,2)$ & $(12,12,256)$ \\
\hline 4 & conv & 512 & $(5,5)$ & $(2,2)$ & $(1,2,1,2)$ & $(6,6,512)$ \\
\hline 5 & dense & $N$ & NA & NA & NA & $(1,1, N)$ \\
\hline 5 & concatenate & NA & NA & NA & NA & $(1,1, N+2 M)$ \\
\hline 6 & dense & 36 & NA & NA & NA & $(1,1,36)$ \\
\hline 7 & reshape & NA & NA & NA & NA & $(6,6,1)$ \\
\hline 8 & conv transpose & 256 & $(5,5)$ & $(2,2)$ & $(1,2,1,2)$ & $(12,12,256)$ \\
\hline 9 & conv transpose & 128 & $(5,5)$ & $(2,2)$ & $(1,2,1,2)$ & $(24,24,128)$ \\
\hline 10 & conv transpose & 64 & $(5,5)$ & $(2,2)$ & $(1,2,1,2)$ & $(48,48,64)$ \\
\hline 11 & conv transpose & 32 & $(5,5)$ & $(2,2)$ & $(1,2,1,2)$ & $(96,96,32)$ \\
\hline 12 & conv transpose & 1 & $(5,5)$ & $(2,2)$ & $(1,2,1,2)$ & $(192,192,1)$ \\
\hline
\end{tabular}

Table S1: Conditional CAE architecture for the mouse-wheel dataset using $N$ latents and $M$ markers (each with an $\mathrm{x}$ and $\mathrm{y}$ value, for a total of $2 M$ marker dimensions). Kernel size and stride size are defined as (x pixels, y pixels); padding size is defined as (left, right, top, bottom); output size is defined as (x pixels, y pixels, channels).

\section{S2.2 Disentangling analysis}

The disentangling analyses presented in Figure 5 require fixing some inputs to the network while varying others. Below we describe this manipulation in more detail. We performed these manipulations on 2-latent networks to make visualization in the latent space easier.

Manipulating markers. We chose a random test frame and varied the $\mathrm{x} / \mathrm{y}$ coordinates for a specific marker (left paw). The limits of the $\mathrm{x} / \mathrm{y}$ values were the $10^{\text {th }}$ (minimum) and $90^{\text {th }}$ (maximum) percentiles of the DGP outputs on the test set for the specified marker. We did not allow different limits for the DLC/DGP networks, in order to make the comparison more direct. After choosing $\mathrm{x} / \mathrm{y}$ values for the specified marker we converted these into a one-hot $2 \mathrm{D}$ array, as with the other (unchanged) markers from the chosen frame. These one-hot 2D arrays were concatenated with the original frame and then fed into the CAE encoder to produce the latents. The latents were then concatenated with the median-subtracted marker values (one of which is being changed, the rest of which stay the same). This vector was then pushed through the decoder network to produce the reconstructions.

Note that in this conditional architecture the latents themselves are marker-dependent, so are not truly held fixed. We also fit conditional CAE architectures where just the decoder was conditioned on the markers, and the encoder only used the frame as input. We found the results from the disentangling analysis to be qualitatively similar, though reconstructions generally looked cleaner with the architectures that incorporated conditioning in both the encoder and decoder networks (data not shown).

Manipulating latents. We chose a random test frame and this time varied the latents while keeping the marker values fixed. Similar to above, we used the $10^{\text {th }}$ (minimum) and $90^{\text {th }}$ (maximum) percentiles of the latents on the test set as limits (this time allowing different limits for each DLC/DGP architecture). We then concatenated the new latent values with the marker values from the original frame, and pushed this vector through the decoder network to produce the reconstructions.

Quantifying disentanglement. To quantify the disentanglement results from Figure 5 (center, right panels), we chose the left paw as a (tracked) target of interest that should ideally not undergo large changes when manipulating the latents. If disentanglement is high (which we desire), the differences between the generated paw and the original paw should be small. For each image generated from the latent manipulation, we take a small crop around the original location of the left paw and compute the MSE between this generated paw and the original paw. 
A

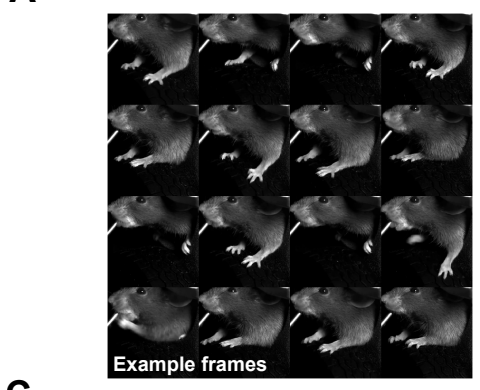

C

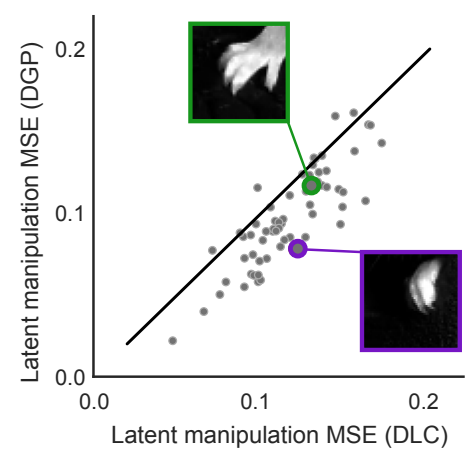

B
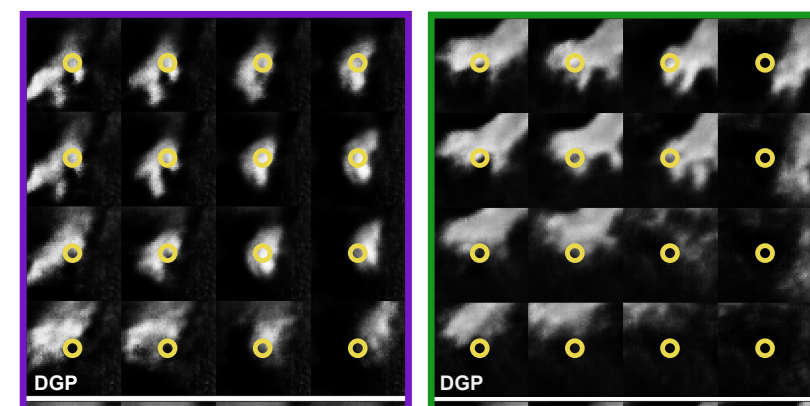

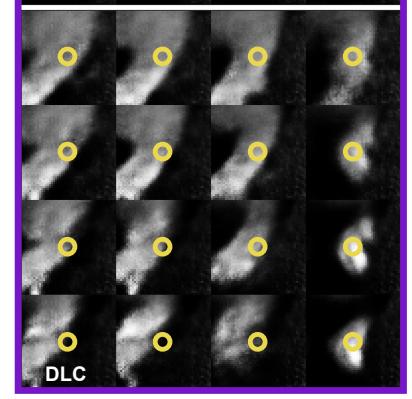

Example 1

Manipulating $A E$ latents Markers fixed

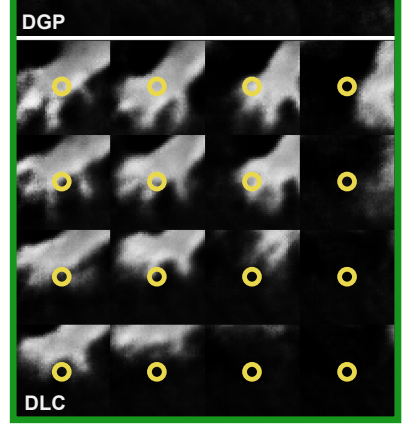

Example 2

Manipulating AE latents Markers fixed

Figure S1: Conditional CAEs display better disentangling properties when using DGP rather than DLC markers. A: A total of 64 representative frames were chosen from the test set by performing k-means on the 2-latent unconditional AE latents; pictured are 16 of those frames, which include the left paw (the target feature of this analysis) in multiple positions. B: For each frame, the markers are held fixed while the 2 latents are varied, producing a matrix of generated images. For each generated image we crop around the left paw. The paw position should remain stable throughout the latent manipulation if there is good disentanglement between latents and markers. Latent manipulations of two example frames are shown (in columns) for networks trained using DGP or DLC markers (in rows). Yellow circles indicate the left paw marker location. C: We compute MSE between each generated frame and the original frame around the paw, and average over all latent values. Small MSE indicates desired stability in the tracked paw. For almost all frames the DGP-trained network exhibits lower MSE, thus demonstrating a higher degree of disentangling.

We repeat this process for an unbiased sample of test frames. To obtain these frames we performed k-means clustering on the unconditional CAE latents. From each of 64 clusters we take the frame that is closest to the cluster centroid (Figure S1A), and perform the process of generating frames by evenly sampling the latent space - 4 grid points along each of 2 dimensions, for a total of 16 generated frames. We compute the MSE in crops around the labeled paw position as described above (Figure S1B, C). We find that on average the CAE-DGP networks have lower MSE, indicating that these disentanglement results generalize to many other paw positions (and therefore marker values) found in this dataset. 


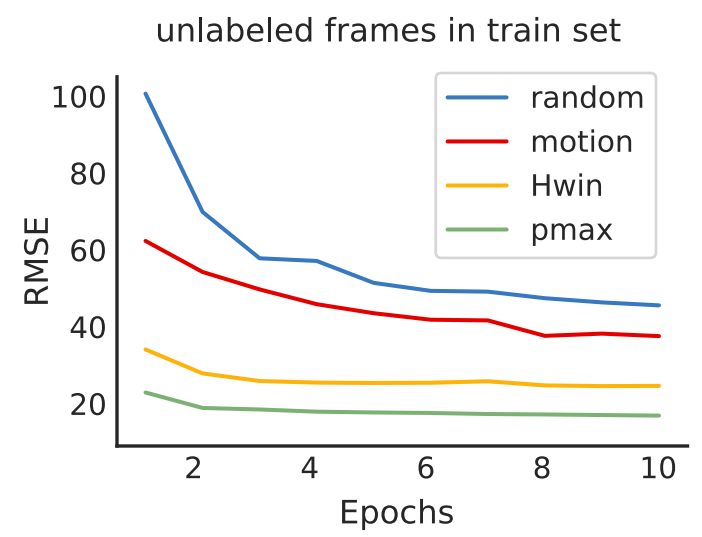

Figure S2: Different active learning strategies can speed up training of DGP without the need to query for new human labels. Evolution of the RMSE during training using four different scoring strategies to query for pseudo targets (unlabeled frames) on the twomice-top-down dataset. The RMSE decays faster by employing active learning strategies to select unlabeled frames to train DGP.

\section{S3 Active learning}

Given a video with a set $N$ of frames, let $M$ be the set of user-labeled frames, and let $U=N-M$ be the set of frames not selected to be labeled. Many previous animal pose estimation algorithms select $M \in N$ by applying $k$-means clustering to a randomly sampled subset of frames in the video, and then uniformly sampling frames from these clusters. The number of clusters for $k$-means is manually set usually as 10 or 25 . Although this approach can work relatively well, several failure modes can occur. For example, if the animals are in fixed positions or not moving for extended periods of time (a common scenario in biological experimental settings), uniformly sampling frames from such a video will result in selecting many identical or redundant frames. Furthermore, if the frames of interest in the video-parts of the video where animals perform behaviors of interest to experimenters-are sparse, randomly sampling frames from a video is highly likely to overlook these frames. Additionally, even if the frames of interest are included during clustering, since these frames are scarce, they may be considered as outliers by a naive $k$-means algorithm and there are no guarantees that these frames of interest would be sampled from any clusters and selected for downstream labeling or training steps.

This problematic lack of diversity in the training set can be addressed by fully supervised algorithms by querying new labeled frames, i.e. by asking the user to manually label new frames based on the performance of the network after training. This process can be repeated several times until the network outputs are satisfactory. Previous work has shown that active learning by querying informative and representative samples can be more effective than passive human labeling [51,52]. Most of this work proposes strategies to score and sample unlabeled frames for manual labeling.

Here we propose to employ these strategies to select not only the set of frames to be manually labeled by an experimenter or oracle $M$, but also to select the subset of unlabeled frames used during training, $S \in U$, which provide additional information to semi-supervised algorithms such as DGP. Unlike semi-supervised learning, which exploits what the learner thinks it knows about the data by employing, for example, unlabeled frames with pseudo targets during training, active learning exploits what the learner does not know about the data, by exploring the space or querying for information about the unlabeled frames [53]. These two approaches can be combined naturally; see [53] for a review.

\begin{tabular}{|c|l|}
\hline Scoring & Description \\
\hline \hline random & Frames are sampled at random from video \\
\hline motion & $\begin{array}{l}\text { Frames are sampled based on their motion energy; frames that are very } \\
\text { similar to their nearest neighbors are sampled less }\end{array}$ \\
\hline$H_{w i n}$ & $\begin{array}{l}\text { Frames are sampled based on the difference between the entropy of mean } \\
\text { predictions and the mean entropy of predictions [54] }\end{array}$ \\
\hline$p_{\max }$ & $\begin{array}{l}\text { Frames are sampled based on their uncertainty; frames with more uncer- } \\
\text { tain network outputs are re-sampled more frequently }\end{array}$ \\
\hline
\end{tabular}

Table S2: Scoring strategies for active learning 
We train DGP using the four different active learning strategies described in Table S2 to query for pseudo targets (unlabeled frames) employed during training. Figure S2 illustrates the evolution of the RMSE for a subset of hidden frames in the training set. We see that with active learning the loss converges faster than by using random sampling. In future work we plan to investigate these different active learning approaches in more detail and across additional datasets.

\section{S4 Results on all datasets}

Figures S3-S6 show the comparisons between DLC and DGP on the mouse-reach, fly-run, twomicetop-down, and fish-swim datasets (Table 1). In each case, results were similar to those seen in Figure 2. Please check these videos to see the trace comparisons between DLC and DGP for these datasets.

We also did some additional experiments with the intermediate models between DLC and DGP, including DLC-ours, DGP-semi and DGP-spatial described in Table S3.

\begin{tabular}{|c|l|}
\hline Model & Description \\
\hline \hline DLC & original DLC implementation, binary target maps for cross entropy loss \\
\hline DLC-ours & our DLC implementation, gaussian target maps for cross entropy loss \\
\hline DGP-semi & DGP model without cliques, semi-supervised loss only \\
\hline DGP-spatial & DGP model with only spatial clique \\
\hline DGP & full DGP model with both spatial and temporal cliques \\
\hline
\end{tabular}

Table S3: All the models we ran for comparison.

Table S4 summarizes the experimental setup for each model and each dataset. Note that the total number of iterations we ran DGP models was way less than the total number of iterations we ran using DLC. For example, we initialized DLC-ours from DLC after 200k; then initialized DGP using DLC-ours after 6k. Equivalently, we can assume that we initialized DGP using DLC after 206k iterations and ran another $80 \mathrm{k}(8 \mathrm{k} \mathrm{x} 10)$ iterations with batch size 1 . Thus, we ran about $280 \mathrm{k}$ iterations in total for DGP while we ran $1 \mathrm{~m}(1,000,000)$ iterations for DLC.

Results are consistent across datasets: DGP and DGP-spatial achieve similar performance across all five datasets, but DGP has smoother traces; each tends to outperform DGP-semi, which in turn outperforms either implementation of DLC. We also provide full videos for the comparisons of 5 traces. 


\begin{tabular}{|c|c|c|c|c|}
\hline Dataset & Model & Initialization & $\begin{array}{l}\text { Number of } \\
\text { iterations }\end{array}$ & Batch size \\
\hline \multirow{5}{*}{ mouse-wheel } & DLC & pre-trained ResNet & $1 \mathrm{~m}$ & 1 \\
\hline & DLC-ours & DLC 200k & $86 \mathrm{k}$ & 1 \\
\hline & DGP-semi & DLC-ours 6k & $8 \mathrm{k}$ & 10 \\
\hline & DGP-spatial & DLC-ours 6k & $8 \mathrm{k}$ & 10 \\
\hline & DGP & DLC-ours 6k & $8 \mathrm{k}$ & 10 \\
\hline \multirow{5}{*}{ mouse-reach } & DLC & pre-trained ResNet & $1 \mathrm{~m}$ & 1 \\
\hline & DLC-ours & DLC $200 \mathrm{k}$ & $31 \mathrm{k}$ & 1 \\
\hline & DGP-semi & DLC-ours 5k & $5 \mathrm{k}$ & 5 \\
\hline & DGP-spatial & DLC-ours 5k & $5 \mathrm{k}$ & 5 \\
\hline & DGP & DLC-ours 5k & $5 \mathrm{k}$ & 5 \\
\hline \multirow{5}{*}{ fly-run } & DLC & pre-trained ResNet & $1 \mathrm{~m}$ & 1 \\
\hline & DLC-ours & DLC $200 \mathrm{k}$ & $185 \mathrm{k}$ & 1 \\
\hline & DGP-semi & DLC-ours 5k & $18 \mathrm{k}$ & 10 \\
\hline & DGP-spatial & DLC-ours 5k & $18 \mathrm{k}$ & 10 \\
\hline & DGP & DLC-ours 5k & $18 \mathrm{k}$ & 10 \\
\hline \multirow{5}{*}{ twomice-top-down } & DLC & pre-trained ResNet & $1 \mathrm{~m}$ & 1 \\
\hline & DLC-ours & DLC $200 \mathrm{k}$ & $114 \mathrm{k}$ & 1 \\
\hline & DGP-semi & DLC-ours $2 \mathrm{k}$ & $12 \mathrm{k}$ & 10 \\
\hline & DGP-spatial & DLC-ours $2 \mathrm{k}$ & $12 \mathrm{k}$ & 10 \\
\hline & DGP & DLC-ours $2 \mathrm{k}$ & $12 \mathrm{k}$ & 10 \\
\hline \multirow{5}{*}{ fish-swim } & DLC & pre-trained ResNet & $1 \mathrm{~m}$ & 1 \\
\hline & DLC-ours & DLC 200k & $170 \mathrm{k}$ & 1 \\
\hline & DGP-semi & DLC-ours $2 \mathrm{k}$ & $17 \mathrm{k}$ & 10 \\
\hline & DGP-spatial & DLC-ours $2 \mathrm{k}$ & $17 \mathrm{k}$ & 10 \\
\hline & DGP & DLC-ours $2 \mathrm{k}$ & $17 \mathrm{k}$ & 10 \\
\hline
\end{tabular}

Table S4: Experimental setup for each dataset and each model. For DLC, we initialized it with a pre-trained ResNet from ImageNet and ran $1 \mathrm{~m}$ iterations using 1 batch stochastic gradient descent (sgd) for all datasets. For the mouse-wheel dataset, we initialized DLC-ours from DLC after 200k iterations and ran it for 86k iterations with batch size 1. We initialized DGP-semi, DGP-spatial, and DGP from DLC-ours after 6k iterations and ran each for another 8k iterations with batch size 10. Likewise, we ran the other four datasets correspondingly. 

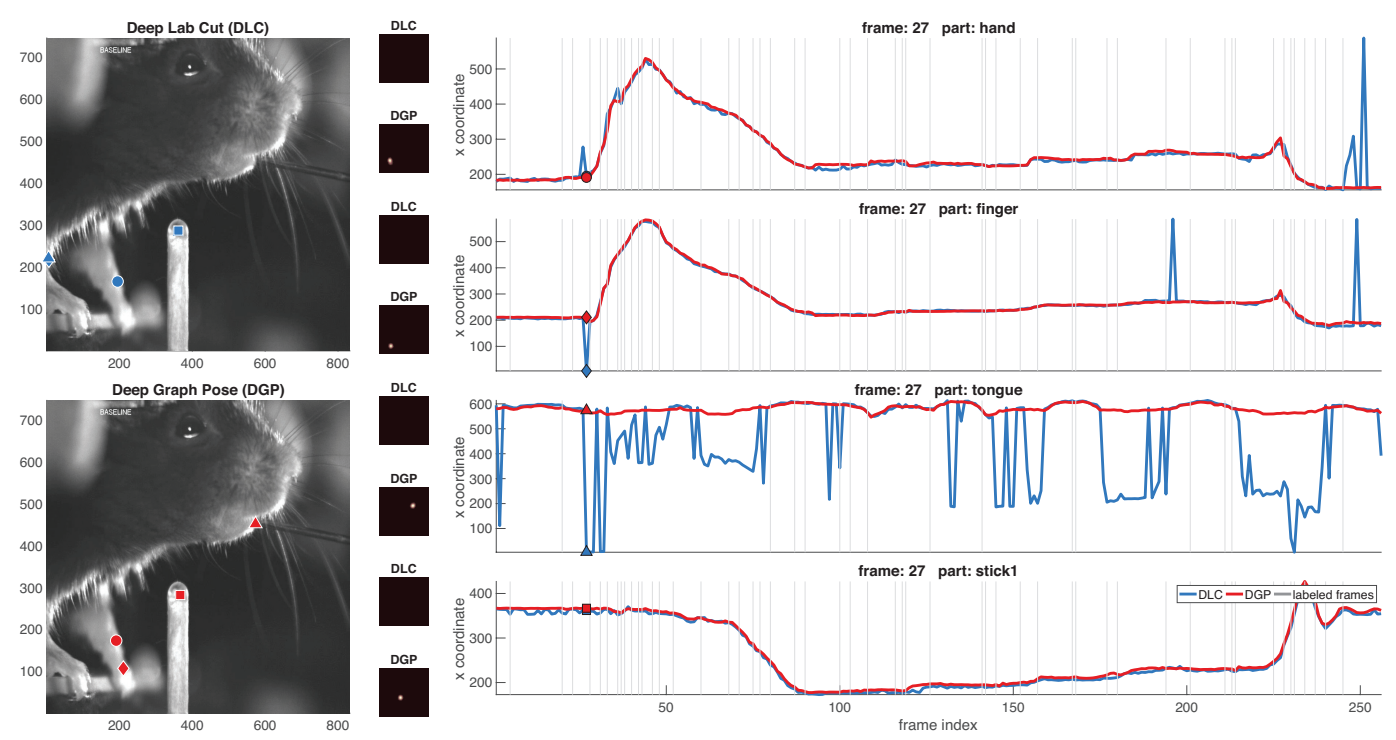

Figure S3: Comparing DeepLabCut (DLC) versus Deep Graph Pose (DGP) on the mouse reaching dataset from [4]. Conventions and conclusions as in Figure 2.
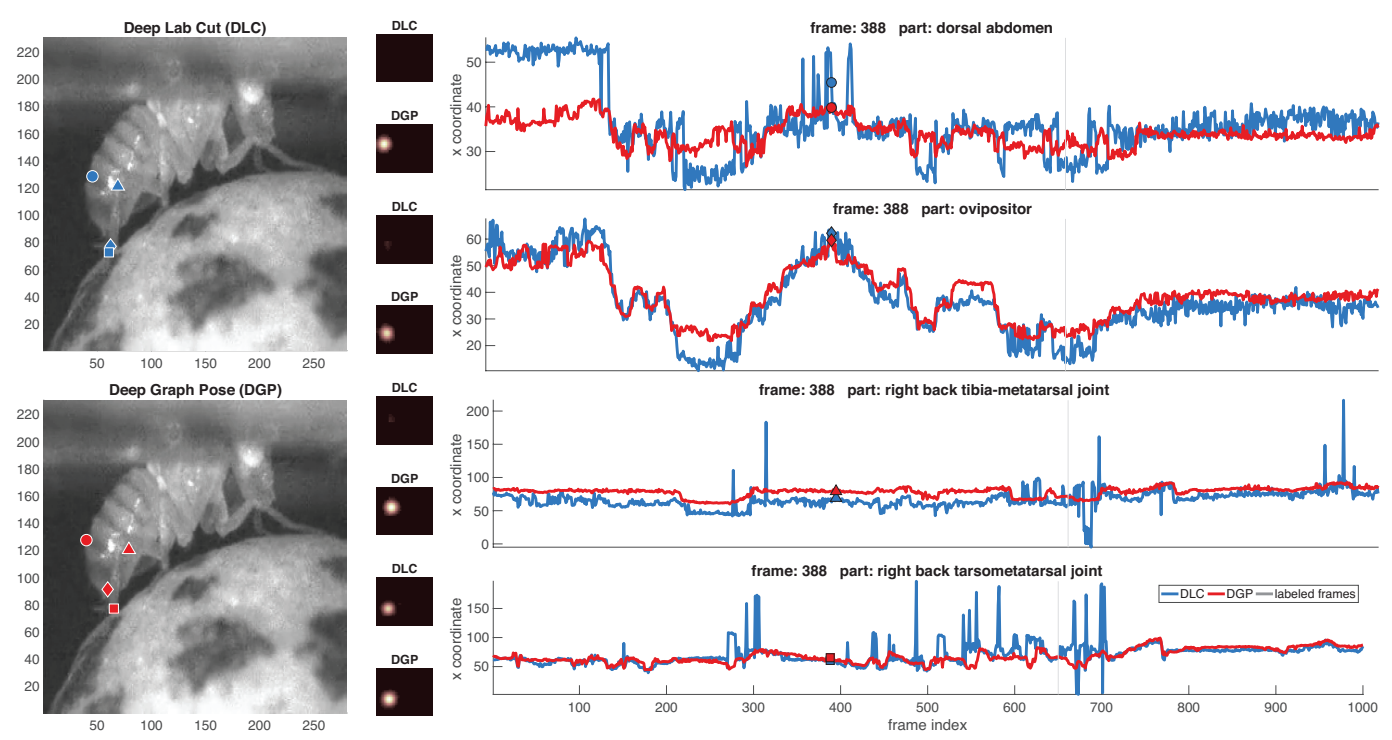

Figure S4: Comparing DeepLabCut (DLC) versus Deep Graph Pose (DGP) on the fly ball-turning dataset from [32]. Conventions and conclusions as in Figure 2. 

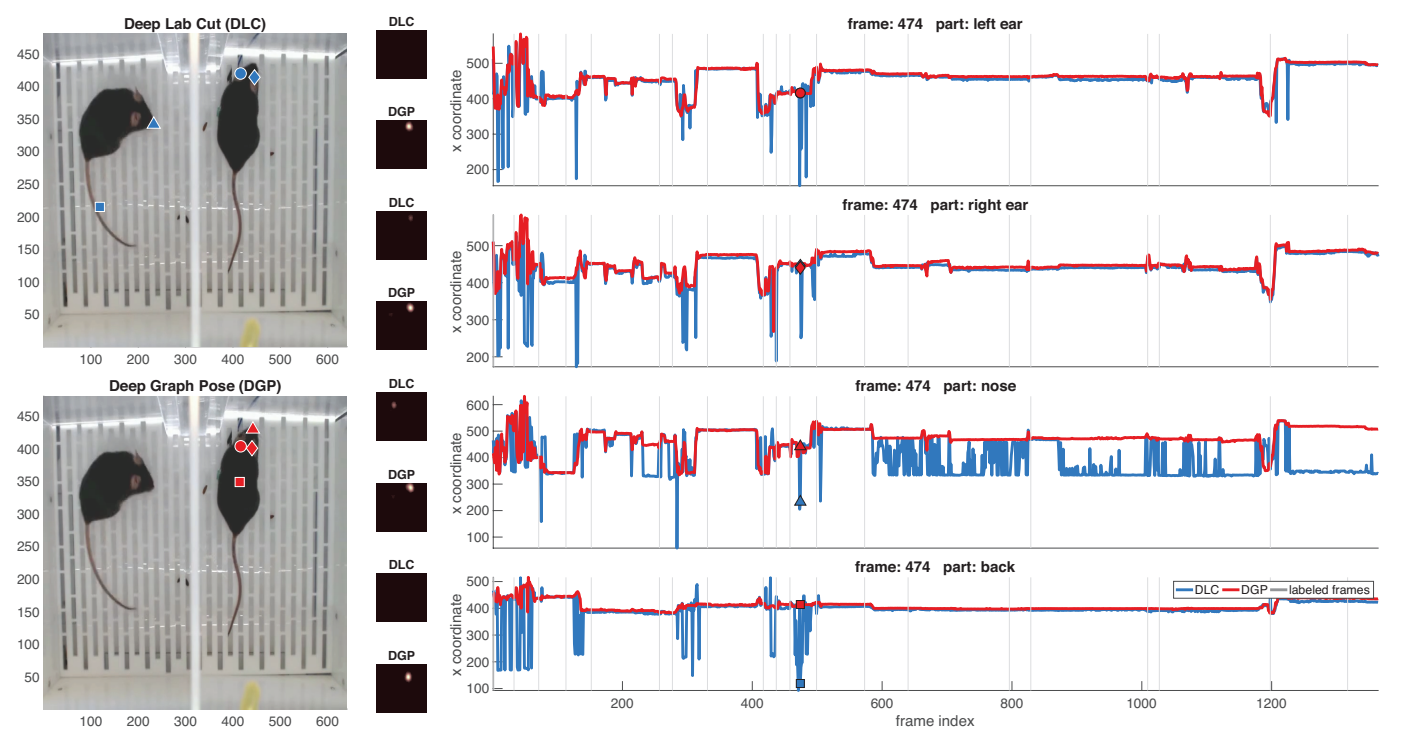

Figure S5: Comparing DeepLabCut (DLC) versus Deep Graph Pose (DGP) on the twomice-top-view dataset with two mice. Conventions and conclusions as in Figure 2.
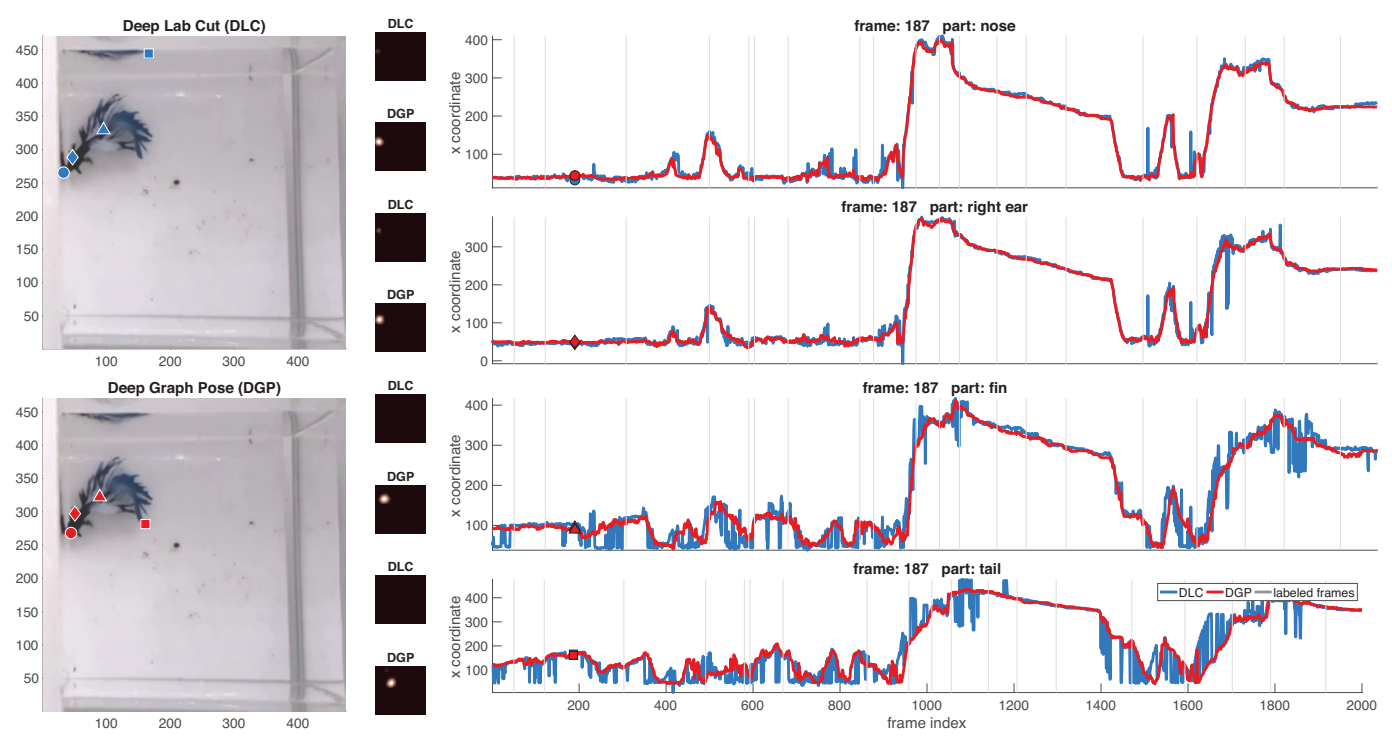

Figure S6: Comparing DeepLabCut (DLC) versus Deep Graph Pose (DGP) on the swimming fish dataset from [33]. Conventions and conclusions as in Figure 2. 